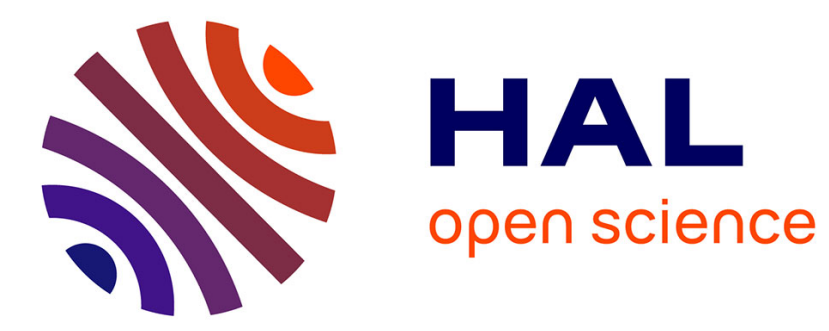

\title{
DATA DEPTH FOR MEASURABLE NOISY RANDOM FUNCTIONS
}

\author{
Stanislav Nagy, Frédéric Ferraty
}

\section{To cite this version:}

Stanislav Nagy, Frédéric Ferraty. DATA DEPTH FOR MEASURABLE NOISY RANDOM FUNCTIONS. Journal of Multivariate Analysis, 2019, 170, pp.95-114. 10.1016/j.jmva.2018.11.003 . hal01980154

\section{HAL Id: hal-01980154 https://hal.science/hal-01980154}

Submitted on 14 Jan 2019

HAL is a multi-disciplinary open access archive for the deposit and dissemination of scientific research documents, whether they are published or not. The documents may come from teaching and research institutions in France or abroad, or from public or private research centers.
L'archive ouverte pluridisciplinaire HAL, est destinée au dépôt et à la diffusion de documents scientifiques de niveau recherche, publiés ou non, émanant des établissements d'enseignement et de recherche français ou étrangers, des laboratoires publics ou privés. 


\title{
DATA DEPTH FOR MEASURABLE NOISY RANDOM FUNCTIONS
}

\author{
STANISLAV NAGY ${ }^{1}$ AND FRÉDÉRIC FERRATY ${ }^{2}$
}

\begin{abstract}
In the literature on data depth applicable to random functions it is usually assumed that the trajectories of all the random curves are continuous, known at each point of the domain, and observed exactly. These assumptions turn out to be unrealistic in practice, as the functions are often observed only at a finite grid of time points, and in the presence of measurement errors. In this work, we provide the necessary theoretical background enabling the extension of the statistical methodology based on data depth to measurable (not necessarily continuous) random functions observed within the latter framework. It is shown that even if the random functions are discontinuous, observed discretely, and contaminated with additive noise, many common depth functionals maintain the fine consistency properties valid in the ideal case of completely observed noiseless functions. For the integrated depth for functions, we provide uniform rates of convergence over the space of integrable functions.
\end{abstract}

\section{INTRODUCTION}

In functional data analysis it is usually assumed that the random curves are observed perfectly, i.e. that the functional values are known precisely, at all points of their domain (also referred to as time), see the monographs of Ramsay and Silverman (2005), Ferraty and Vieu (2006), or Horváth and Kokoszka (2012), or the recent surveys of Cuevas (2014) and Goia and Vieu (2016). While this assumption is appropriate for the development of basic methodology for dealing with functions, in practice it is seldom satisfied. On the contrary, real functional datasets are never observed continuously in time, and often a substantial amount of additive noise, introduced by the measurement procedure, prevents from observing these values accurately. If the functions are observed densely enough to permit the reconstruction of their original trajectories, data analysis is routinely performed after a preprocessing step. Here the discrete, inaccurately observed values are smoothed to approximate the unobservable, supposedly regular function. Nonetheless, such data imputation may disturb the correctness of the subsequent statistical analysis. In the present contribution, we consider the field of statistical depth for functional data, and provide theoretical justification for the use of pre-smoothed functional data in combination with the analysis based on depth.

Statistical depth is a nonparametric instrument applicable to multivariate (and infinitedimensional) data. For these, it aims to introduce a generalization of quantiles. By identifying points in the sample space located "centrally" with respect to a given probability distribution $P$, it is capable of distinguishing "typical" and "potentially outlying" observations by assigning to them very high, or very low values of depth, respectively. For a general account on data depth in the classical setup of finite-dimensional spaces see Zuo and Serfling (2000), and the references therein.

\footnotetext{
${ }^{1}$ Charles University, Department of Probability and Math. Statistics, Czech Republic

${ }^{2}$ Toulouse Mathematics Institute, University of Toulouse, France

E-mail addresses: nagy@karlin.mff.cuni.cz.

Date: January 14, 2019.
} 
The first extension of depth to functional data analysis is due to Fraiman and Muniz (2001). Since then, numerous depth functionals have been proposed, mostly in the space of continuous functions over a compact interval. Let us mention the work of Cuevas and Fraiman (2009), López-Pintado and Romo (2009, 2011), Mosler (2013), Claeskens et al. (2014), Chakraborty and Chaudhuri (2014) and a recent article of Narisetty and Nair (2016). Most of these diverse approaches can be classified into three groups of depths following similar ideas, as discussed in Gijbels and Nagy (2015) and Nagy et al. (2016b): (i) integrated depths, (ii) band depths, and (iii) infimal depths.

In some fields of functional data analysis, noisy random functions have already been studied intensively; a body of literature on this topic encompassing functional linear regression, functional principal components, continuous additive models, etc. can be found in Yao et al. (2005), Crambes et al. (2009), Paul and Peng (2009), Jiang and Wang (2010), Li and Hsing (2010), Wu et al. (2010), Müller et al. (2013), Radchenko et al. (2015), Hsing and Eubank (2015) and Zhang and Wang (2016), among others. In those works, noisy functional data are typically either (i) pre-smoothed in the first step, and then statistical analysis is performed for the smoothed approximants of the data curves; or (ii) the statistical procedure in question is revisited, and suitably applied directly to the available discrete noisy observations. The latter approach is usually less versatile, and requires a specific type of data (such as random curves in Hilbert spaces decomposable into their functional principal components). In this paper, we thus follow the former method, and explore its potential in the field of functional data depth.

For functional data depth, no theoretical results of this kind are available. Though, also here the pre-smoothing step is very common, and known to give good results in practice, see Cuevas et al. (2006, 2007), Hyndman and Shang (2010), Sun and Genton (2011, 2012), López-Pintado and Wei (2011), Ieva and Paganoni (2013), López-Pintado et al. (2014), Claeskens et al. (2014) and Narisetty and Nair (2016). In the present paper we bridge the concepts of noisy functional data and data depth, and provide theoretical properties of depth computed from pre-smoothed functions. To this end, we focus on the main categories of depth functionals (integrated depths, band depths, and infimal depths), and establish the mathematical background necessary for depth-based analysis of discretely observed and contaminated curves.

In Section 2 we gather the preliminaries and notation. Then, the first result establishing mild conditions for the weak convergence of the empirical measures based on the reconstructions of the noisy curves is given. This is a non-trivial extension of the classical theorem of Varadarajan (1958) to functional data. Considering noisy (hence discontinuous) curves, it is necessary to abandon the framework of depths defined in the space of continuous functions. In Section 3, extensions of the main representatives of integrated and band depths into the space of integrable functions are given, and their properties are examined. Some new consistency results for these are provided. In particular, it is shown that under suitable conditions both integrated, and (adjusted) band depths retain the fine consistency properties valid in the space of perfectly observed continuous functions. In Section 4 we focus on integrated depths. For the first time, we provide the rate of convergence of a depth functional over the whole functional space. Using an extension of the theory from Section 2 we derive the rate of convergence of the integrated depth in the setup of noisy random functions, and demonstrate that if the noisy curves are observed densely in time, then the same rate of convergence as for perfectly observed data can be achieved. Some interesting extensions of our theory are discussed in Section 5. A 
simulation study illustrating the performance of the proposed methodology is given in Section 6. Its complete results can be found in the Supplementary Material accompanying the paper. The proofs are provided in the Appendix.

\section{Weak Convergence for Noisy Functional Data}

Let $(\Omega, \mathcal{F}, \mathrm{P})$ be the probability space on which all random elements are defined. For a measurable space $S, \mathcal{P}(S)$ is the set of all probability measures defined on $S$, and $X \sim P \in \mathcal{P}(S)$ stands for a random variable $X$ in $S$ with distribution $P$. For $P \in \mathcal{P}(S)$, $\omega \in \Omega$ and $n \geq 1, P_{n}(\omega)=P_{n} \in \mathcal{P}(S)$ denotes the empirical measure of the random sample $X_{1}, \ldots, X_{n}$ from $P$ corresponding to $\omega$. The weak convergence of a sequence $\left\{P_{\nu}\right\}_{\nu=1}^{\infty} \subset \mathcal{P}(S)$ to $P \in \mathcal{P}(S)$ is denoted by $P_{\nu} \underset{\nu \rightarrow \infty}{\stackrel{w}{\longrightarrow}} P$. The space of continuous functions over $[0,1]$ is denoted by $\mathcal{C}([0,1]) . L^{2}([0,1])$ is the space of measurable functions $x:[0,1] \rightarrow \mathbb{R}$ for which $x^{2}$ is Lebesgue integrable. For $\lambda$ the Lebesgue measure on $\mathbb{R}$, the norm of $x \in L^{2}([0,1])$ is denoted by

$$
\|x\|:=\sqrt{\int_{0}^{1} x(t)^{2} \mathrm{~d} \lambda(t) .}
$$

For $X \sim P \in \mathcal{P}\left(L^{2}([0,1])\right), P_{t} \in \mathcal{P}(\mathbb{R})$ stands for the marginal distribution of $X(t)$ for $t \in[0,1]$, and $F_{t}$ for its distribution function. The marginal distribution of the empirical measure $P_{n}$ at $t \in[0,1]$ is denoted by $P_{n, t} \in \mathcal{P}(\mathbb{R})$, and its distribution function by $F_{n, t}$.

Consider a random function $X \sim P \in \mathcal{P}\left(L^{2}([0,1])\right)$, that is a mapping

$$
X:[0,1] \times \Omega \rightarrow \mathbb{R}:(t, \omega) \mapsto X(t, \omega), \quad(\mathcal{B}([0,1]) \times \mathcal{F}) \text {-measurable },
$$

such that $X(\cdot, \omega) \in L^{2}([0,1])$ for P-almost all $\omega \in \Omega$. $\mathcal{B}([0,1])$ stands for the Borel sets in $[0,1]$. Note that we assume that $X$ is measurable with respect to the product $\sigma$-algebra. This technical assumption is needed to assure proper measurability of all the considered quantities, especially with respect to the integrated depth for discontinuous functions (see Nagy, 2017a). When not necessary, the argument of the random element $\omega$ in $X$ will be omitted.

Let $X_{1}, \ldots, X_{n}$ be independent random functions distributed as $X$. Assume that the complete set of functional values $\left\{X_{i}(t): t \in[0,1]\right\}, i=1, \ldots, n$, cannot be observed directly. Instead, for each $i$, only the finite vector

$$
\left(X_{i, 1}^{*}, \ldots, X_{i, m_{i}}^{*}\right)=\left(X_{i}\left(T_{i, 1}\right)+\varepsilon_{i, 1}, \ldots, X_{i}\left(T_{i, m_{i}}\right)+\varepsilon_{i, m_{i}}\right) \in \mathbb{R}^{m_{i}},
$$

is known to the observer. Here, $m_{i} \geq 1$ is an integer and $\left(T_{i, 1}, \ldots, T_{i, m_{i}}\right) \in[0,1]^{m_{i}}$ is a random sample of size $m_{i}$ from a distribution $T \sim P_{T} \in \mathcal{P}([0,1]) . \varepsilon_{i, 1}, \ldots, \varepsilon_{i, m_{i}}$ are independent, centred random variables such that $\operatorname{Var} \varepsilon_{i, j}=\sigma^{2}\left(T_{i, j}\right)$, where $\sigma^{2}:[0,1] \rightarrow$ $[0, \infty)$ is a deterministic function. In what follows, all the variables $\varepsilon_{i, j}, T_{i, j}$ and $X_{i}$ are assumed to be independent; a more general scenario will be discussed in Section 5 . The choice $\sigma^{2} \equiv 0$ covers the case when the random functions are observed discretely without noise. This setup was considered by Nagy et al. (2016a) in the space $\mathcal{C}([0,1])$, and in the present contribution we extend these results substantially to discontinuous functions contaminated with measurement errors, using very different proof techniques.

Though the number of the observed functional values $m_{i}$ in (2) can be considered fixed for a single function $X_{i}$, it will typically grow as the sampling process from $P$ continues, i.e. $m_{i} \underset{i \rightarrow \infty}{\longrightarrow} \infty$. Random functions observed within this setup are in the literature often 
called dense functional data ( $\mathrm{Li}$ and Hsing, 2010), in contrast to sparse functional data (Yao et al., 2005), where $m_{i}$ is bounded as $i$ grows.

The assumption of having a non-random $m_{i}$ in $(2)$ is made for mere notational convenience. In what follows $m_{i}$ could be replaced by its stochastic counterpart, and where applicable, $m_{i} \underset{i \rightarrow \infty}{\longrightarrow} \infty$ can always be replaced by $m_{i} \underset{i \rightarrow \infty}{\stackrel{\text { a.s. }}{\longrightarrow}} \infty$ for $m_{i}$ independent of the other random variables. Likewise, it is possible to consider a related model, where in the random sample $X_{1}, \ldots, X_{n}$ the function $X_{i}$ is observed at $m_{i, n}$ points. In this case, one can proceed analogously as pursued here, the only assumption being $m_{i, n} \geq m_{i}$ for all $i \geq 1$ and $n \geq i$, for some $m_{i} \underset{i \rightarrow \infty}{\longrightarrow} \infty$. Note that in (2) we do not have to require the usual $m_{i, n} \underset{n \rightarrow \infty}{\longrightarrow} \infty$ for all $i \geq 1$. That is, in our setup, for $i \geq 1$ fixed the $i$ th sampled function is not bound to be observed at more points as the sampling process continues. Therefore, the currently developed theory naturally embeds also the commonly considered setup of increasingly dense functional data (where $\min _{i=1, \ldots, n} m_{i, n} \underset{n \rightarrow \infty}{\longrightarrow} \infty$ ).

For the random vector (2), a reconstruction of the unobserved curve $X_{i} \in L^{2}([0,1])$ based on these points is necessary in order to perform statistical inference on the original distribution $P$. This is often done by nonparametric smoothing. Here, we mainly focus on the kernel estimator of $X_{i}$ (for other smoothing methods see Section 5) - for a kernel $K: \mathbb{R} \rightarrow[0, \infty)$ and a bandwidth $h_{m_{i}}>0$, we approximate $X_{i}$ by

$$
\tilde{X}_{i}(t):=\frac{\sum_{j=1}^{m_{i}} X_{i, j}^{*} K\left(\left(t-T_{i, j}\right) / h_{m_{i}}\right)}{\sum_{j=1}^{m_{i}} K\left(\left(t-T_{i, j}\right) / h_{m_{i}}\right)} \quad \text { for } t \in[0,1] .
$$

After all the curves $X_{i}$ are approximated by $\widetilde{X}_{i}, P_{n} \in \mathcal{P}\left(L^{2}([0,1])\right)$ based on $X_{1}, \ldots, X_{n}$ can be estimated by the measure of empirical type $\widetilde{P}_{n} \in \mathcal{P}\left(L^{2}([0,1])\right)$ concentrated in the smoothed functions $\widetilde{X}_{1}, \ldots, \widetilde{X}_{n}$. In the following theorem we formulate a weak convergence result for $\widetilde{P}_{n}$. For this, we need the following assumptions:

(A1) $\mathrm{E} X(T)^{2}<\infty$.

(A2) There exist constants $0<r \leq R$ and $0<b<B$ such that for the kernel $K$

$$
B \mathbb{I}[-R \leq u \leq R] \geq K(u) \geq b \mathbb{I}[-r \leq u \leq r] \quad \text { for all } u \in \mathbb{R} .
$$

(A3) $P_{T}$ has a density over $[0,1]$ bounded from below by $c_{T}>0$.

(A4) $\sup _{t \in[0,1]} \sigma^{2}(t) \leq \sigma^{2}$ for some $\sigma^{2}<\infty$.

(A5) $h_{m_{i}} \underset{i \rightarrow \infty}{\longrightarrow} 0$ and $m_{i} h_{m_{i}} \underset{i \rightarrow \infty}{\longrightarrow} \infty$.

Theorem 1. Under the assumptions $(A 1)-(A 5), \mathrm{P}\left(\widetilde{P}_{n} \underset{n \rightarrow \infty}{\stackrel{w}{\longrightarrow}} P\right)=1$, that is the sequence of empirical measures $\left\{\widetilde{P}_{n}(\omega)\right\}_{n=1}^{\infty}$ converges to $P$ weakly in $\mathcal{P}\left(L^{2}([0,1])\right)$ for P-almost all $\omega \in \Omega$.

As will be seen in Section 3 below, the Varadarajan's type of result displayed in Theorem 1 can be directly applied to the problem of consistency of depth functionals. In Section 4, under slightly stricter assumptions imposed on $X$, the rates of convergence of the sequence of approximating functions $\left\{\widetilde{X}_{n}\right\}_{n=1}^{\infty}$ to the sequence of independent realisations of $X$ are investigated. As such, these results provide a quantitative version of Theorem 1. 


\section{Depth Functionals in $L^{2}([0,1])$}

In the literature on depth for functional data, numerous approaches to depth computation have been proposed. Though most of these are constructed in the setup of continuous random functions (that is for $x, X \in \mathcal{C}([0,1])$ ), their extensions to the space of measurable functions are rather straightforward. We begin by defining rigorously some representatives of the most widely studied depth functionals, extended to the space $L^{2}([0,1])$. These include a general univariate integrated depth (Nagy et al., 2016b), the $h$-mode depth, and the adjusted band depth, the latter two taking the form of band depths for functions (Gijbels and Nagy, 2015).

Many other depths follow the ideas behind these two general classes of functionals. Namely, the original band depths (López-Pintado and Romo, 2009), the corrected band depths (López-Pintado and Jornsten, 2007), the sparse band depths (López-Pintado and Wei, 2011), the local band depths (Agostinelli and Romanazzi, 2011), various versions of multivariate band depths (Ieva and Paganoni, 2013, López-Pintado et al., 2014), the halfregion depths (López-Pintado and Romo, 2011), the set band depths (Whitaker et al., 2013), and other related functionals all fall logically into the setup of general band depths for functional data. On the other hand, the so-called modified versions of all these band depths, the modified volume depth (Genton et al., 2014), and the integrated depths of Cuevas and Fraiman (2009) and Claeskens et al. (2014) are representatives of depths of integrated type. Theoretical properties of all these functionals in $L^{2}([0,1])$ can be derived from the results given in the present section in an analogous manner. For band depths, however, adjustment as proposed by Gijbels and Nagy (2015) is necessary to be introduced into the depth evaluation to avoid consistency issues.

In the definitions below, assume that the depth of the function $x \in L^{2}([0,1])$ with respect to (w.r.t.) the distribution of the random function $X \sim P \in \mathcal{P}\left(L^{2}([0,1])\right)$ is to be determined. We start with a generic definition of a depth functional of integrated type based on a univariate depth.

Definition (Fraiman and Muniz, 2001, Cuevas and Fraiman, 2009, Nagy et al., 2016b). The integrated depth of $x$ w.r.t. $P$ is given by

$$
a D(x ; P):=\int_{0}^{1} D_{1}\left(x(t) ; P_{t}\right) \mathrm{d} \lambda(t) .
$$

As for the choice of the univariate depth in the definition of $a D$, the only assumptions we make are that $D_{1}: \mathbb{R} \times \mathcal{P}(\mathbb{R}) \rightarrow[0,1]:(u, Q) \mapsto D_{1}(u ; Q)$ is a (depth) mapping that is jointly measurable in its domain, weakly continuous in the distribution argument, and universally consistent (see Nagy et al., 2016b, Sections 4.6 and 5.2). The latter two conditions mean that $D_{1}$ satisfies $\sup _{u \in \mathbb{R}}\left|D_{1}\left(u ; Q_{\nu}\right)-D_{1}(u ; Q)\right| \underset{\nu \rightarrow \infty}{\longrightarrow} 0$ whenever $Q_{\nu} \underset{\nu \rightarrow \infty}{\stackrel{w}{\longrightarrow}} Q$ in $\mathcal{P}(\mathbb{R})$ such that $Q$ is absolutely continuous, and that $\sup _{u \in \mathbb{R}} \mid D_{1}\left(u ; Q_{n}\right)-$ $D_{1}(u ; Q) \mid \underset{n \rightarrow \infty}{\stackrel{\text { a.s. }}{\longrightarrow}} 0$ for any $Q \in \mathcal{P}(\mathbb{R})$ and the sequence of the associated empirical measures $\left\{Q_{n}\right\}_{n=1}^{\infty}$. It is easy to show that these conditions are obeyed for most univariate depths. In particular, writing $F_{Q}$ for the distribution function of $Q \in \mathcal{P}(\mathbb{R})$, they are 
true (Nagy et al., 2016b, Appendix A) for any of

$$
\begin{aligned}
& D_{1}^{a}(u ; Q):=1 / 2-\left|1 / 2-F_{Q}(u)\right|, \\
& D_{1}^{b}(u ; Q):=\min \left\{F_{Q}(u), 1-\lim _{v \rightarrow u-} F_{Q}(v)\right\}, \\
& D_{1}^{c}(u ; Q):=F_{Q}(u)\left(1-\lim _{v \rightarrow u-} F_{Q}(v)\right), \\
& D_{1}^{d}(u ; Q):=\frac{1}{J-1} \sum_{j=2}^{J} \mathrm{P}\left(u \in\left[\min _{i=1, \ldots, j} U_{i}, \max _{i=1, \ldots, j} U_{i}\right]\right),
\end{aligned}
$$

where in the last expression $J \geq 2$ and $U_{1}, \ldots, U_{J}$ is a random sample from $Q$. The depth $D_{1}^{a}$ was introduced into (4) by Fraiman and Muniz (2001). If $F_{Q}$ is continuous it is equivalent with $D_{1}^{b}$, the well-known halfspace depth (Tukey, 1975) in $\mathbb{R}$. The depth $D_{1}^{c}$ relates to the simplicial depth (Liu, 1990) in $\mathbb{R}$. In (4) it was used by Cuevas and Fraiman (2009). $D_{1}^{d}$ forms in (4) the modified band depth for functional data (López-Pintado and Romo, 2009). For $J=2, D_{1}^{d}$ is equivalent with $D_{1}^{c}$.

In the definitions of band depths that follow, $K_{D}:[0, \infty) \rightarrow[0, \infty)$ is a continuous function such that $\lim _{t \rightarrow \infty} K_{D}(t)=0$. It plays the role of a smoothing kernel, and can differ from the kernel $K$ utilized to recover the random functions in Section 2.

Definition (Cuevas et al., 2006, 2007). The $h$-mode depth of $x$ w.r.t. $P$ is given by

$$
h D(x ; P):=\frac{1}{h(P)} \mathrm{E} K_{D}\left(\frac{\|x-X\|}{h(P)}\right),
$$

where $h: \mathcal{P}\left(L^{2}([0,1])\right) \rightarrow(0, \infty)$ is a bandwidth such that $h\left(P_{\nu}\right) \underset{\nu \rightarrow \infty}{\longrightarrow} h(P)$ whenever $P_{\nu} \underset{\nu \rightarrow \infty}{\stackrel{w}{\longrightarrow}} P$ in $\mathcal{P}\left(L^{2}([0,1])\right)$.

Definition (Gijbels and Nagy, 2015). The adjusted band depth of order $J \geq 1$ of $x$ w.r.t. $P$ is given by

$$
b D(x ; P):=\operatorname{E} K_{D}\left(d\left(x, B\left(X_{1}, \ldots, X_{J}\right)\right)\right),
$$

where $X_{1}, \ldots, X_{J}$ are independent random functions from $P$,

$$
\begin{aligned}
B\left(x_{1}, \ldots, x_{J}\right):= & \bigcup_{\substack{N \subset[0,1] \\
\lambda(N)=1}}\left\{y \in L^{2}([0,1]): \min _{j=1, \ldots, J} x_{j}(t) \leq y(t) \leq \max _{j=1, \ldots, J} x_{j}(t)\right\} \\
& =\left\{y \in L^{2}([0,1]): \min _{j=1, \ldots, J} x_{j} \leq y \leq \max _{j=1, \ldots, J} x_{j} \lambda \text {-almost everywhere }\right\}
\end{aligned}
$$

is the band of functions $x_{1}, \ldots, x_{J} \in L^{2}([0,1])$ and

$$
d\left(x, B\left(x_{1}, \ldots, x_{J}\right)\right):=\inf \left\{\|x-y\|: y \in B\left(x_{1}, \ldots, x_{J}\right)\right\}
$$

is the distance between $x$ and the band of functions.

The depth $b D$ for $J=1$ is the same as $h D$ for the choice $h(P)=1$ for all $P \in$ $\mathcal{P}\left(L^{2}([0,1])\right)$. The sample versions of all the defined depths are formed by substituting the population measure $P$ by the empirical measure $P_{n}$ in (4), (6), and (7), respectively, if $P_{n}$ is observed. If the complete curves are not available, the empirical measure of their reconstructions $\widetilde{P}_{n}$ is used. Note that in the list of depths applicable to integrable 
functions we omitted any version of the infimal type of depth functionals (Mosler and Polyakova, 2012, Gijbels and Nagy, 2015) such as the basic infimal depth for functions (Mosler, 2013)

$$
i D(x ; P):=\inf _{t \in[0,1]} D_{1}\left(x(t) ; P_{t}\right),
$$

for $x \in L^{2}([0,1]), P \in \mathcal{P}\left(L^{2}([0,1])\right)$ and $D_{1}$ as for $a D$. Other representative of infimal depths that can be found in the literature is the functional halfspace depth discussed in Dutta et al. (2011). Also, the extremal depth (Narisetty and Nair, 2016) is based on a related idea. These depths are not defined well for $x \in L^{2}([0,1])$, since the functional values of $x$ in (8) are given uniquely only for almost every $t \in[0,1]$. More importantly, even if the definition of the depth was modified accordingly, it can be shown that these functionals suffer from serious consistency issues in the case of discontinuous observations. To illustrate this, consider the example of $X \sim P \in \mathcal{P}\left(L^{2}([0,1])\right)$ defined such that the functional value $X(0)$ is standard normally distributed, and $X(t)=W(t)$ for $t>0$, where $W$ is a standard Wiener process on $[0,1]$, independent of $X(0)$. For this random function, say $D_{1}=D_{1}^{a}$, and $x \equiv 0$, we get $D_{1}\left(x(t) ; P_{t}\right)=1 / 2$ for all $t \in[0,1]$. Nonetheless, for any finite random sample from $P$ and its empirical measure $P_{n}$, there exists a subset $S$ of $[0,1]$ of positive Lebesgue measure such that $D_{1}\left(x(t) ; P_{n, t}\right)=0$ for all $t \in S$ (see Gijbels and Nagy, 2015, Example 5). Thus, neither the depth $i D$, nor its version with an essential infimum in (8), can be consistent in $L^{2}([0,1])$. Furthermore, the consistency difficulties of the infimal depths can be shown to persist also for smoothed versions of the functional data, as can be seen by obvious modifications of Nagy et al. (2016a, Example 5). These results are true even though all the marginal distributions $P_{t}$ are absolutely continuous, which is sufficient for the consistency of $i D$ in $\mathcal{C}([0,1])$ (Gijbels and Nagy, 2015, Theorem 5).

The next theorem deals with the sample version consistency of all the considered depth functionals (except from $i D$ ) in $L^{2}([0,1])$ in the case when the random functions are observed completely (that is when $P_{n}$ is observable). It provides a unified extension of several consistency results scattered in the literature (see, for instance, Nagy, 2017b).

Theorem 2. Let $D$ be one of the functional depths $a D, h D$ or $b D$. For any $P \in$ $\mathcal{P}\left(L^{2}([0,1])\right)$

$$
\sup _{x \in L^{2}([0,1])}\left|D\left(x ; P_{n}\right)-D(x ; P)\right| \underset{n \rightarrow \infty}{\stackrel{\text { a.s. }}{\longrightarrow}} 0
$$

If the distribution of $X \sim P$ is such that $X$ is $\mathrm{P}$-almost surely continuous over $[0,1]$, also the supremum norm (equivalent with the $L^{\infty}([0,1])$ norm)

$$
\|x\|_{\infty}:=\sup _{t \in[0,1]}|x(t)| \quad \text { for } x \in \mathcal{C}([0,1])
$$

can be used in both the $h$-mode and the adjusted band depth to evaluate the depth of functions in $\mathcal{C}([0,1])$. A proof analogous to that of Theorem 2 can be used to show that in this case both $h D$ and $b D$ are universally consistent over $\mathcal{C}([0,1])$. In Section 6 we use both $L^{2}([0,1])$ and $\mathcal{C}([0,1])$ versions of these two depths to assess their performance when the sample functions are approximated.

While in Theorem 2 we were able to state the universal consistency results without any distributional assumptions, in what follows for $a D$ it is necessary to make one additional assumption: 
(A6) The marginal distributions $P_{t}$ of $P \in \mathcal{P}\left(L^{2}([0,1])\right)$ are absolutely continuous for all $t \in[0,1]$.

With (A6) we are able to formulate the consistency result also for depths based on noisy observations. This theorem substantially improves on the related consistency results for discretely observed noiseless random functions in $\mathcal{C}([0,1])$ provided in Nagy et al. (2016a).

Theorem 3. Let $P \in \mathcal{P}\left(L^{2}([0,1])\right)$. Then

$$
\sup _{x \in L^{2}([0,1])}\left|D\left(x ; \widetilde{P}_{n}\right)-D(x ; P)\right| \underset{n \rightarrow \infty}{\stackrel{\text { a.s. }}{\longrightarrow}} 0
$$

if (A1)-(A5) are satisfied and $D$ is $h D$ or bD. Moreover, if also (A6) is true, then the result holds true also for $D=a D$.

To see that (A6) cannot be dropped for $a D$, consider $X \sim P \in \mathcal{P}\left(L^{2}([0,1])\right)$ with $\mathrm{P}(X \equiv 0)=1, K(u)=\mathbb{I}[|u| \leq 1]$, and $P_{T}$ the uniform distribution on $[0,1]$. Then, using* $D_{1}^{b}, D_{1}^{c}$ or $D_{1}^{d}$ for $D_{1}$ in (4) one gets for $x \equiv 0$ that $a D(x ; P)=1$. One the other hand, for $\widetilde{P}_{n}$ based on kernel smoothed noisy random functions (with positive noise variance) we have by the usual (univariate) central limit theorem that

$$
\lim _{i \rightarrow \infty} \mathrm{P}\left(\tilde{X}_{i}(t)<0\right)=\lim _{i \rightarrow \infty} \mathrm{P}\left(\tilde{X}_{i}(t)>0\right)=1 / 2 \quad \text { for all } t \in[0,1],
$$

and thus for P-almost all $\omega \in \Omega$ we have $\lim \sup _{n \rightarrow \infty} a D\left(x ; \widetilde{P}_{n}(\omega)\right)<1=a D(x ; P)$.

\section{Rates of Convergence}

In the present section we focus on the integrated depth $a D$ based on the univariate depth $D_{1}=D_{1}^{a}$ from (5). It constitutes the simplest representative of integrated depths for functions. Extensions of the present results for other univariate depth functions $D_{1}$ as considered in Section 3 are possible using analogous techniques. Initially, we state the uniform rate of convergence of the integrated depth over $L^{2}([0,1])$. As far as we are aware, it is the first result of this kind for a depth constructed for functional data.

Theorem 4. For any $P \in \mathcal{P}\left(L^{2}([0,1])\right)$

$$
\sup _{x \in L^{2}([0,1])}\left|a D\left(x ; P_{n}\right)-a D(x ; P)\right|=\mathcal{O}_{P}\left(n^{-1 / 2}\right) .
$$

4.1. Rate of Convergence for Smoothed Random Functions. Now, a theorem concerning the rates of convergence of the reconstructed random curves towards the noiseless random functions will be stated. For this, a refinement of the condition (A1) is necessary in what follows.

(A1 $\left.{ }^{*}\right)$ There exist constants $M, L>0$ and $\beta \in(0,1]$ such that

$$
\begin{gathered}
\sup _{t \in[0,1]} \mathrm{E} X(t)^{2} \leq M, \quad \text { and } \\
\mathrm{P}\left(|X(s)-X(t)| \leq L|s-t|^{\beta} \text { for all } s, t \in[0,1]\right)=1 .
\end{gathered}
$$

${ }^{*}$ The depth $D_{1}^{a}(\cdot ; Q)$ is not well suited for $Q \in \mathcal{P}(\mathbb{R})$ whose distribution function is discontinuous; for instance, for $Q$ supported in a singleton $D_{1}^{a}(u ; Q)=0$ for all $u \in \mathbb{R}$. 
Theorem 5. Let conditions $\left(A 1^{*}\right)$, (A2)-(A5) be satisfied. Then there exists a constant $c>0$ such that

$$
\mathrm{E}\left\|X_{i}-\tilde{X}_{i}\right\|^{2} \leq c \frac{\sigma^{2}+M}{m_{i} h_{m_{i}}}+L^{2} h_{m_{i}}^{2 \beta} .
$$

As a corollary of Theorem 5 we obtain that for the optimal choice of bandwidth in our setup (see Györfi et al., 2002)

$$
h_{m_{i}}=\mathcal{O}\left(m_{i}^{-1 /(2 \beta+1)}\right)
$$

we have for some $C>0$

$$
\mathrm{E}\left\|X_{i}-\tilde{X}_{i}\right\| \leq \sqrt{\mathrm{E}\left\|X_{i}-\tilde{X}_{i}\right\|^{2}} \leq C m_{i}^{-\beta /(2 \beta+1)} \quad \text { for all } i \geq 1 .
$$

This is the optimal rate of convergence attainable in the nonparametric regression setting as derived by Stone (1982) for $\beta \leq 1$, see also the discussion in Kohler et al. (2009). In the sequel, for simplicity we restrict ourselves only to the case of the optimal choice of bandwidths (10). Though, all the computations are straightforward to be adapted for a general sequence $\left\{h_{m_{i}}\right\}_{i=1}^{\infty}$.

4.2. Rate of Convergence of Depth for Noisy Functions. Recall that for a random function $X \sim P \in \mathcal{P}\left(L^{2}([0,1])\right)$ and $t \in[0,1], F_{t}$ stands for the distribution function of the univariate random variable $X(t)$. Assume that $\left(\mathrm{A} 1^{*}\right)$ and $(\mathrm{A} 6)$ are satisfied for $P$. Then we may proceed as in the proof of Gijbels and Nagy (2015, Theorem 4) and show that the collection of all marginal distribution functions $\left\{F_{t}: t \in[0,1]\right\}$ of $P$ is in fact a collection of uniformly equicontinuous functions, i.e.

$$
\lim _{\varepsilon \rightarrow 0+} \sup _{t \in[0,1]} \sup _{\left|s-s^{\prime}\right| \leq \varepsilon}\left|F_{t}(s)-F_{t}\left(s^{\prime}\right)\right|=0 .
$$

Therefore, it is possible to find a deterministic, continuous and non-decreasing function $\delta_{F}:[0, \infty) \rightarrow[0, \infty)$ depending only on $P$ such that $\delta_{F}(0)=0$, and

$$
\sup _{t \in[0,1]} \sup _{\left|s-s^{\prime}\right| \leq \varepsilon}\left|F_{t}(s)-F_{t}\left(s^{\prime}\right)\right| \leq \delta_{F}(\varepsilon) \quad \text { for } \varepsilon>0 .
$$

The function $\delta_{F}$ is called a uniform modulus of continuity of the collection $\left\{F_{t}: t \in[0,1]\right\}$.

In what follows, we need to manipulate with the Legendre transform of the function $g(\nu):=\delta_{F}(1 / \nu), \nu>0$. Recall that for $g$ its Legendre transform $g^{*}$ is a function defined by

$$
g^{*}(\mu):=\sup _{\nu>0}(\nu \mu-g(\nu))
$$

for $\mu \in \mathbb{R}$ such that the right hand side is finite. Now, we are ready to state the main result of the contribution - the uniform rate of convergence of the integrated depth for noisy random functions.

Theorem 6. Let $X \sim P \in \mathcal{P}\left(L^{2}([0,1])\right)$ be such that $\left(A 1^{*}\right)$, (A2)-(A6) are satisfied.

(i) Then

$$
\sup _{x \in L^{2}([0,1])}\left|a D\left(x ; \widetilde{P}_{n}\right)-a D(x ; P)\right|=\mathcal{O}_{P}\left(\max \left\{-g^{*}\left(-\frac{1}{n} \sum_{i=1}^{n} m_{i}^{-\beta /(2 \beta+1)}\right), n^{-1 / 2}\right\}\right) .
$$


(ii) If $\delta_{F}(\varepsilon)=K \varepsilon^{\alpha}$ for some $\alpha \in(0,1]$ and $K>0$, then

$$
\sup _{x \in L^{2}([0,1])}\left|a D\left(x ; \widetilde{P}_{n}\right)-a D(x ; P)\right|=\mathcal{O}_{P}\left(\left(\frac{1}{n} \sum_{i=1}^{n} m_{i}^{-\beta /(2 \beta+1)}\right)^{\alpha /(1+\alpha)}\right) .
$$

(iii) If, furthermore, $m_{n} \sim n^{r}$ for some $r>0$, then

$$
\sup _{x \in L^{2}([0,1])}\left|a D\left(x ; \widetilde{P}_{n}\right)-a D(x ; P)\right|= \begin{cases}\mathcal{O}_{P}\left(n^{-r \alpha \beta /((1+\alpha)(2 \beta+1))}\right) & \text { if } r<(2 \beta+1) / \beta, \\ \mathcal{O}_{P}\left((\log (n) / n)^{\alpha /(1+\alpha)}\right) & \text { if } r=(2 \beta+1) / \beta, \\ \mathcal{O}_{P}\left(n^{-\alpha /(1+\alpha)}\right) & \text { if } r>(2 \beta+1) / \beta .\end{cases}
$$

The rate in part (iii) of Theorem 6 is the optimal rate $\mathcal{O}_{P}\left(n^{-1 / 2}\right)$ from Theorem 4 if $\alpha=1$ and $r>(2 \beta+1) / \beta$, in all other cases it is slower. For Lipschitz continuous random functions $(\beta=1)$ this corresponds to $r>3$. So, if $m_{n}$ is much larger than $n$, then the contamination does not degrade the rate of convergence; in other words, the high-dimensional setting (i.e. $m_{n} \gg n$ ) is a blessing in the contaminated functional data problem. In part (iii) of Theorem 6 we consider only polynomial rate of convergence for $m_{n}$; analogous results can be easily obtained under different assumptions. In that case, it is possible to see that if $m_{n}$ converges fast and the distribution of $P$ is smooth enough $(\alpha=1)$, the oracle rate of convergence from Theorem 4 is attainable also for the reconstructed curves based on observations contaminated by noise.

\section{General Approximation Results}

In Sections 2, 3 and 4, our attention was focused on the analysis using the standard Nadaraya-Watson kernel smoother (3), under the assumptions of independence of the error terms $\varepsilon_{i, 1}, \ldots, \varepsilon_{i, m_{i}}$, and the random design of the observation times $T_{i, j}$ in (2). Though, our main results are not restricted to this setting. Indeed, it is not difficult to generalise our findings to many other scenarios, such as those concerning (i) other nonparametric smoothing methods, and (ii) approximation setups with possibly fixed observation points, or correlated errors terms.

Similarly as in Section 2, let $\widetilde{X}_{i}$ be an approximant of the unobserved functional datum $X_{i}$ based on some information available about $X_{i}$. Denote by $\widetilde{P}_{n} \in \mathcal{P}\left(L^{2}([0,1])\right)$ the probability measure concentrated in the smoothed functions $\widetilde{X}_{1}, \ldots, \widetilde{X}_{n}$, corresponding to a random sample $X_{1}, \ldots, X_{n}$. The following general version of Theorems 1,3 and 6 can be devised.

Theorem 7. Let $X_{1}, X_{2}, \ldots$ be independent random functions distributed as $X \sim P \in$ $\mathcal{P}\left(L^{2}([0,1])\right)$. Suppose that

$$
a_{n}:=\mathrm{E}\left\|X_{n}-\widetilde{X}_{n}\right\|=o(1) \quad \text { as } n \rightarrow \infty .
$$

Then $\mathrm{P}\left(\widetilde{P}_{n} \underset{n \rightarrow \infty}{\stackrel{w}{\longrightarrow}} P\right)=1$, and

$$
\sup _{x \in L^{2}([0,1])}\left|D\left(x ; \widetilde{P}_{n}\right)-D(x ; P)\right| \underset{n \rightarrow \infty}{\stackrel{\text { a.s. }}{\longrightarrow}} 0
$$


for $D$ either $h D$ or $b D$. If, moreover, the random function $X$ is $\mathrm{P}$-almost surely continuous over $[0,1]$ and $(A 6)$ is true, then

$$
\sup _{x \in L^{2}([0,1])}\left|a D\left(x ; \widetilde{P}_{n}\right)-a D(x ; P)\right|=\mathcal{O}_{P}\left(\max \left\{-g^{*}\left(-\frac{1}{n} \sum_{i=1}^{n} a_{i}\right), n^{-1 / 2}\right\}\right) .
$$

Finally, if also $\delta_{F}(\varepsilon)=K \varepsilon^{\alpha}$ for some $\alpha \in(0,1]$ and $K>0$, then

$$
\sup _{x \in L^{2}([0,1])}\left|a D\left(x ; \widetilde{P}_{n}\right)-a D(x ; P)\right|=\mathcal{O}_{P}\left(\left(\frac{1}{n} \sum_{i=1}^{n} a_{i}\right)^{\alpha /(1+\alpha)}\right) .
$$

The proof of Theorem 7 is omitted, as it follows closely the derivations in the proofs of Theorems 1, 3 and 6 in the Appendix.

Under sets of assumptions similar to our conditions (A1)-(A6) for the NadarayaWatson kernel smoother, condition (14) can be verified for many other nonparametric smoothing methods. For instance, for the local polynomial fitting procedure and independent errors in model (2), formula (14) can be obtained from the theory provided in Fan and Gijbels (1996). Likewise, if the points $T_{i, j}$ in (2) are not chosen randomly, wellestablished results in fixed-design nonparametric regression problems provide conditions under which (14) can be verified. If additional smoothness assumptions, such as the existence and boundedness of a higher-order derivative of the random function $X$ can be guaranteed P-almost surely, rates of convergence faster than those in Theorem 5 can be found using Theorem 7.

Finally, some extensions of our theory may be obtained also in the situation when the error terms $\varepsilon_{i, 1}, \ldots, \varepsilon_{i, m_{i}}$ are allowed to be dependent in (2). Then, one deals with the problem of nonparametric regression with correlated errors. Under suitable assumptions on the correlation structure of the random errors, also in that case condition (14) can be recovered. For a general account of results of this type we refer to Liu (2001), Opsomer et al. (2001), or a recent paper of De Brabanter et al. (2018).

\section{Simulation Study}

We complete this paper by illustrating the performance of the proposed method on a small simulation study. It constitutes part of a larger study, including various choices of error variances, and an infinite-dimensional model for the random functions. The complete results of the study, including all its source codes, can be found in the Supplementary Material. The study is performed in R 3.4.2 (R Core Team, 2015) using efficient Fortran implementations for kernel smoothing and functional depth computation, developed by the authors. Most of these functions are freely available as part of $\mathrm{R}$ package ddalpha (Pokotylo et al., 2017).

Consider the model for densely observed functional data as described in Section 2, in combination with the functional depths introduced in Section 3. Our main aim is to compare possibilities for evaluating depth of discretely observed functional data, and to identify scenarios under which the depth based on complete realisations of functions is well approximated by the depth based on the reconstructions of these. The random variable $X \sim P \in \mathcal{P}\left(L^{2}([0,1])\right)$ is given by

$$
X(t)=(5 t)^{2}(3 / 4-t)+A \cos (B \pi t) / 2+C \sin (D \pi t / 3) \quad \text { for } t \in[0,1],
$$


where $A, B, C$ and $D$ are independent standard Gaussian random variables. Consider a random sample $X_{1}, \ldots, X_{n}$ from $P$ of size $n=50$. For the sake of computer processing, in $\mathrm{R}$, the complete function $X_{i}$ is represented by a vector of size $d=1001$ of its functional values taken at $d$ equidistant points in $[0,1]$.

In the analysis, $X_{i}$ is assumed to be observed discretely at $m_{i}=\lceil 50+i / 5\rceil$ independent random observation points as discussed in Section 2. These are distributed according to

(T1) the uniform distribution over $[0,1]$;

(T2) the beta distribution with parameters 2 and 2; and

(T3) the beta distribution with parameters 0.7 and 0.9 .

In setup (T1) the observation points are spread almost regularly over the domain. This makes the reconstruction of $X_{i}$ rather simple, at least for larger values of $m_{i}$. In (T2) the majority of the observed points concentrates in the centre of the domain, while in (T3) mostly points near the boundaries of the interval are given.

For the noise variance we consider three different scenarios

(V1) $\sigma_{1}^{2}(t)=\tau^{2}$

(V2) $\sigma_{2}^{2}(t)=\tau^{2}\left(1+t^{2}\right)^{2}$; and

(V3) $\sigma_{3}^{2}(t)=\tau^{2} \mathbb{I}\left[t \in\left[\min \left\{U_{1}, U_{2}\right\}, \max \left\{U_{1}, U_{2}\right\}\right]\right]$, where $U_{1}$ and $U_{2}$ are two independent uniformly distributed random variables in $[0,1]$, different for each $X_{i}$.

The constant $\tau$ is in this section always set to $\tau=1 / 2$. In the extended study we consider also the case $\tau=1 / 4$. Scenarios (V1) and (V2) correspond to typical homoscedastic and heteroscedastic noise, respectively. (V3) represents the situation when the noise level varies with the sampling process, and each function $X_{i}$ is contaminated in a different subset of its domain. Note that in scenario (V3) the variance of the noise term $\sigma_{3}^{2}(t)$ is random, and therefore it technically does not satisfy condition (A4). Though, it is easy to see that for a random quantity $\sigma^{2}(t)$ a condition like $\operatorname{E} \sup _{t \in[0,1]} \sigma^{2}(t) \leq \sigma^{2}<\infty$ is enough for all the presented results to hold true. In the short simulation study presented here we consider only the situation when the error terms $\varepsilon_{i, j}$ in (2) are independent; in the Supplementary Material we provide also some results for the case of correlated errors.

In each of the scenarios above, the sample curves are reconstructed using five methods:

(S1) constant only approximation

$$
\widetilde{X}_{i}(t)=\frac{1}{m_{i}} \sum_{j=1}^{m_{i}} X_{i}^{*}\left(T_{i, j}\right) \quad \text { for all } t \in[0,1]
$$

denoting $X_{i}^{*}\left(T_{i, j}\right)=X_{i, j}^{*}$

(S2) piecewise linear interpolation, that is

$$
\tilde{X}_{i}(t)=X_{i}^{*}\left(T_{i,(j)}\right)+\frac{t-T_{i,(j)}}{T_{i,(j+1)}-T_{i,(j)}}\left(X_{i}^{*}\left(T_{i,(j+1)}\right)-X_{i}^{*}\left(T_{i,(j)}\right)\right) \quad \text { for } t \in\left[T_{i,(j)}, T_{i,(j+1)}\right),
$$

where $0=T_{i,(0)}, 1=T_{i,\left(m_{i}+1\right)}, T_{i,(1)}, \ldots, T_{i,\left(m_{i}\right)}$ is the ordered sample of the observation points of $X_{i}, X_{i}^{*}\left(T_{i,(0)}\right)=X_{i}^{*}\left(T_{i,(1)}\right)$ and $X_{i}^{*}\left(T_{i,\left(m_{i}+1\right)}\right)=X_{i}^{*}\left(T_{i,\left(m_{i}\right)}\right)$;

(S3) kernel smoothing described in detail in Section 2, with the Gaussian kernel

$$
K(u)=(2 \pi)^{-1 / 2} \exp \left(-u^{2} / 2\right) ;
$$

(S4) local linear smoothing (Fan and Gijbels, 1996), with the Gaussian kernel (15); and 
$(\mathrm{T} 1)$
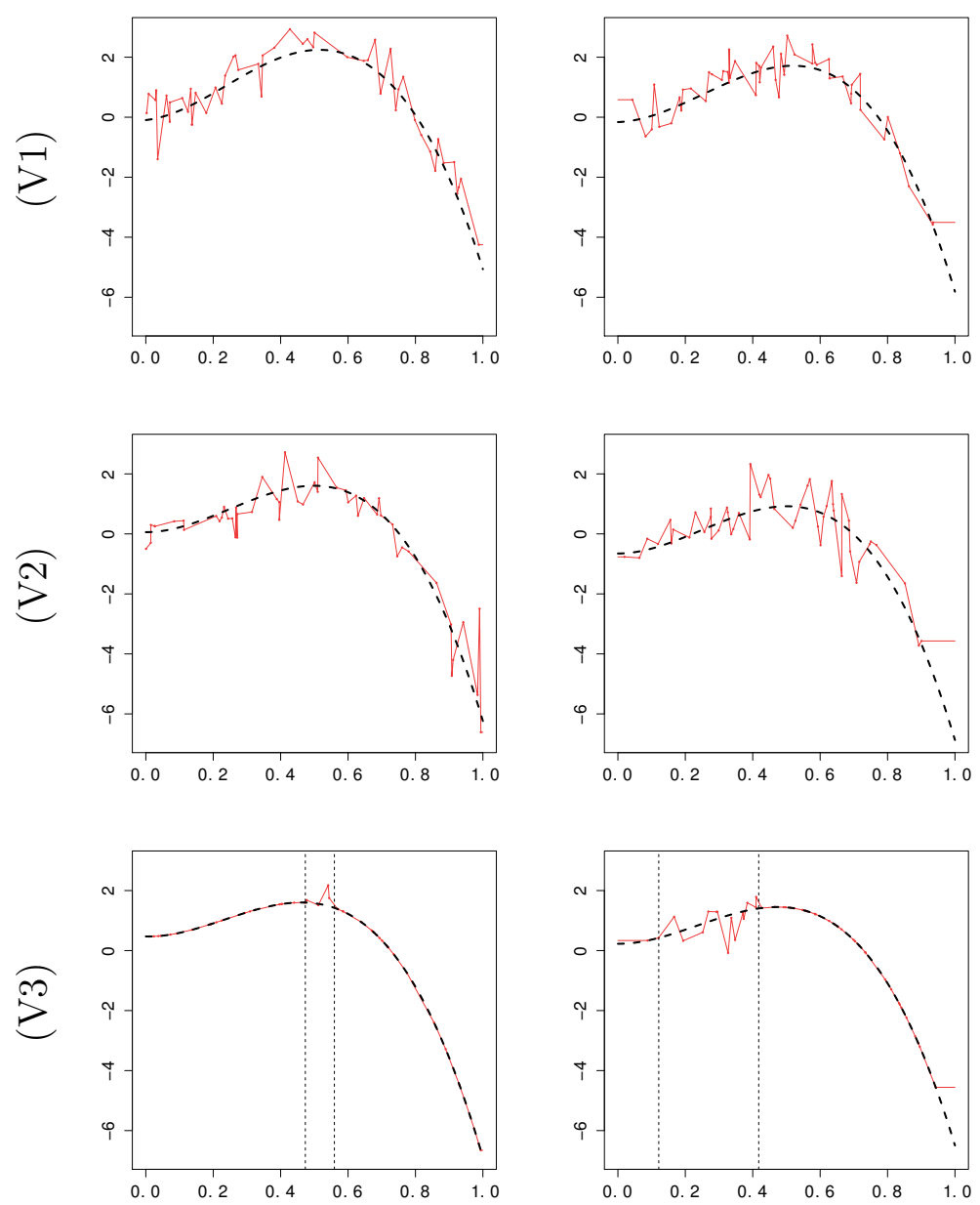

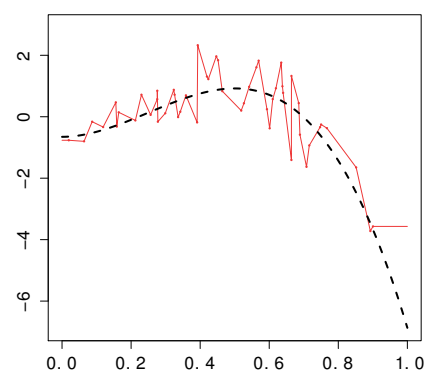

$(\mathrm{T} 2)$

(T3)
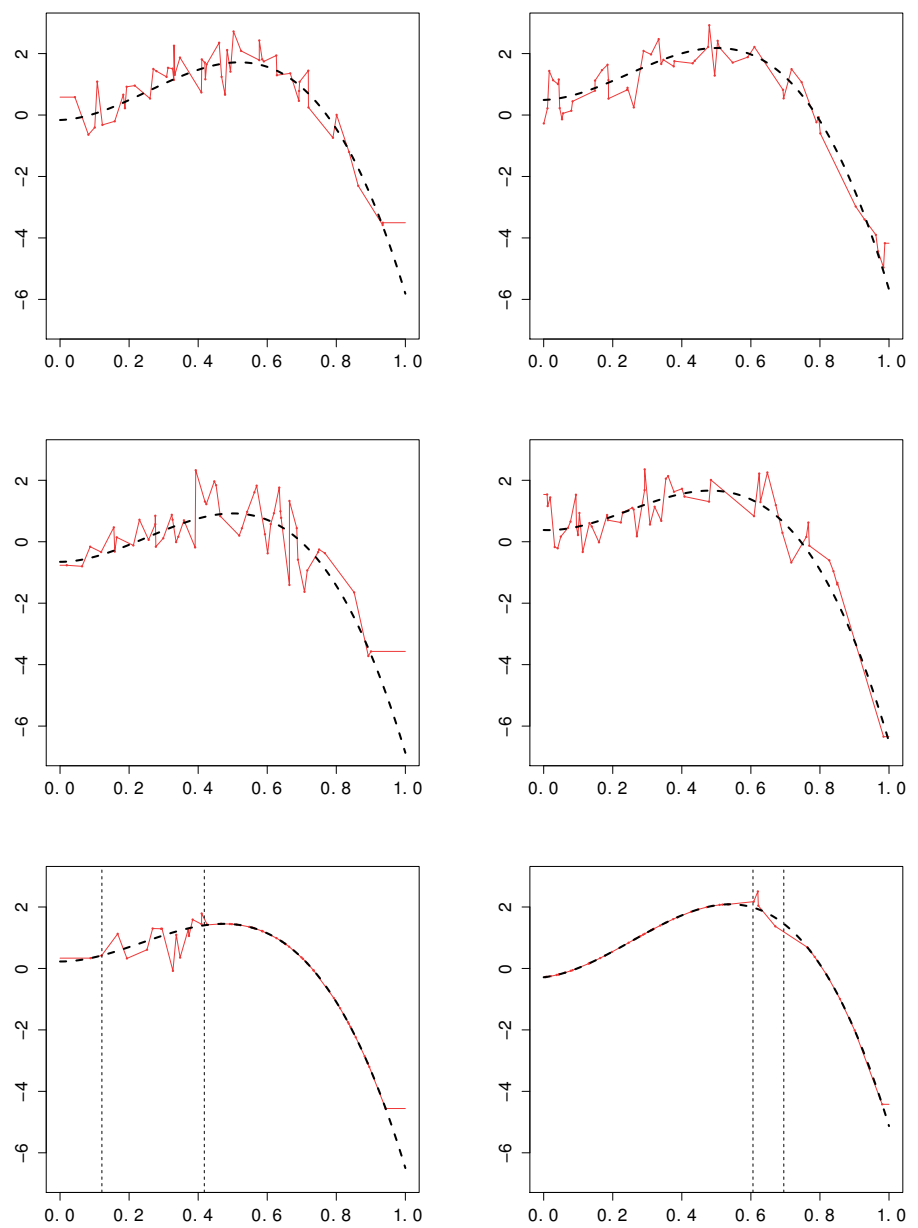

FIGURE 1. For each of the scenarios (T1)-(T3), (V1)-(V3), a single random function $X_{i}$ (thick dashed), and its piecewise linear reconstruction (S2) based on the corresponding $m_{i}=50$ discrete contaminated observations (thin solid) are displayed. For (V3), the vertical dashed lines represent the realised values of the variables $U_{1}$ and $U_{2}$ controlling the noise level.

(S5) local linear smoothing in the presence of correlated errors, described in De Brabanter et al. (2018, Section 5), based on a consecutive application of a bimodal kernel function $\bar{K}(u)=2 u^{2} \exp \left(-u^{2}\right) / \sqrt{\pi}$ and a Gaussian kernel $K(u)$ from (15). Method (S1) is used only as a benchmark - the results for the remaining methods should be compared taking into account the performance of this most naive reconstruction method. Method (S5) is an adaptation of the local linear smoother (S4) that is capable of dealing with correlated errors. Here, it is used mainly for comparison with (S4); for some simulations where method (S5) bears great relevance see the Supplementary Material.

In (S3)-(S5) the bandwidths are taken to be different for each function $X_{i}$. In (S3) and (S4) they are selected using the standard leave-one-out cross-validation. For (S5) the complete description of the bandwidth selection procedure can be found in De Brabanter et al. (2018). All these bandwidth selection methods typically provide sufficiently accurate results, in combination with an acceptable computational cost. A fast version of the 
kernel smoothing method (S3) is taken from the R package ddalpha (Pokotylo et al., 2017); the fast local polynomial smoothers (S4) and (S5) implemented in R package locpol (Cabrera, 2012) are considered. For a single random function from models (T1)(T3), (V1)-(V3), and its piecewise linear interpolation (S2) based on the corresponding contaminated points see Figure 1.

Five depth functionals are considered in the simulations:

$a D$ : the integrated depth using the univariate depth $D_{1}^{a}$ from (5);

$h D_{1}$ : the $h$-mode depth with the $L^{2}([0,1])$ norm $(1)$, the Gaussian kernel $K_{D}$ as in (15) and $h\left(\widetilde{P}_{n}\right)$ chosen as the 20th percentile of the distribution of $\left\|\widetilde{X}_{i}-\widetilde{X}_{j}\right\|, i \neq j$ (in accordance with the choice of Cuevas et al., 2007);

$h D_{2}$ : the $h$-mode depth with the supremum norm (9), kernel (15), and bandwidth as for $h D_{1}$;

$b D_{1}$ : the adjusted band depth with the $L^{2}([0,1])$ norm $(1), J=2$ and $K_{D}(u)=$ $\exp (-u)$

$b D_{2}$ : the adjusted band depth with the supremum norm $(9), J=2$ and $K_{D}(u)=$ $\exp (-u)$.

In the comparison, these depths are computed with respect to the empirical measure $P_{n}$ based on the original (noiseless) curves $X_{1}, \ldots, X_{n}$, and then also with respect to the empirical measure $\widetilde{P}_{n}$ based on the reconstructed curves $\widetilde{X}_{1}, \ldots, \widetilde{X}_{n}$. A reasonable reconstruction method should provide a good approximation of the depth values $D\left(X_{i} ; P_{n}\right)$ for each $i$.

\begin{tabular}{|c|c|c|c|c|c|c|}
\hline & & (S1) & $(\mathrm{S} 2)$ & (S3) & $(\mathrm{S} 4)$ & (S5) \\
\hline \multirow{4}{*}{ (V1) } & $a D$ & $0.602(0.094)$ & $0.890(0.026)$ & $0.932(0.018)$ & $0.940(0.017)$ & $0.936(0.020)$ \\
\hline & $h D_{1}$ & $0.585(0.108)$ & $0.898(0.029)$ & $0.922(0.026)$ & $0.933(0.034)$ & $0.937(0.030)$ \\
\hline & $h D_{2}$ & $0.522(0.113)$ & $0.788(0.065)$ & $0.761(0.074)$ & $0.748(0.081)$ & $0.783(0.068)$ \\
\hline & $b D_{1}$ & $0.758(0.110)$ & $0.960(0.020)$ & $0.966(0.018)$ & $0.960(0.056)$ & $0.965(0.048)$ \\
\hline \multirow{5}{*}{$(\mathrm{V} 2)$} & $b D_{2}$ & $0.630(0.104)$ & $0.850(0.054)$ & $0.825(0.061)$ & $0.796(0.074)$ & $0.829(0.063)$ \\
\hline & $a \bar{D}$ & $\overline{0} . \overline{5} \overline{9} 5(0 . \overline{104})$ & $0 . \overline{8} 36(0.04 \overline{4})$ & $\overline{0.90} \overline{0} \overline{(0.027)}$ & $0 . \overline{9} 10(\overline{0} . \overline{0} 6)$ & $\overline{0.90 \overline{2}}(0.0 \overline{2} 9)$ \\
\hline & $h D_{1}$ & $0.599(0.107)$ & $0.832(0.044)$ & $0.870(0.041)$ & $0.880(0.042)$ & $0.889(0.036)$ \\
\hline & $h D_{2}$ & $0.539(0.113)$ & $0.615(0.107)$ & $0.640(0.097)$ & $0.617(0.103)$ & $0.668(0.096)$ \\
\hline & $b D_{1}$ & $0.766(0.100)$ & $0.916(0.037)$ & $0.935(0.032)$ & $0.932(0.045)$ & $0.941(0.028)$ \\
\hline \multirow{2}{*}{---} & $b D_{2}$ & $0.640(0.105)$ & $0.686(0.094)$ & $0.718(0.082)$ & $0.692(0.091)$ & $0.741(0.083)$ \\
\hline & $\overline{a D}$ & $\overline{0} . \overline{6} \overline{0} \overline{(0.103)}$ & $0 . \overline{9} 6 \overline{3}(0.012)$ & $\overline{0.9 \overline{7}} \overline{(0.009)}$ & $\overline{0} . \overline{9} \overline{7} \overline{(0.008)}$ & $0 . \overline{9} \overline{4}(0.009)$ \\
\hline \multirow{4}{*}{ (V3) } & $h D_{1}$ & $0.604(0.104)$ & $0.934(0.024)$ & $0.954(0.021)$ & $0.972(0.016)$ & $0.979(0.008)$ \\
\hline & $h D_{2}$ & $0.537(0.112)$ & $0.707(0.072)$ & $0.766(0.083)$ & $0.845(0.077)$ & $0.912(0.044)$ \\
\hline & $b D_{1}$ & $0.768(0.088)$ & $0.977(0.011)$ & $0.983(0.009)$ & $0.987(0.015)$ & $0.989(0.014)$ \\
\hline & $b D_{2}$ & $0.637(0.094)$ & $0.703(0.063)$ & $0.829(0.065)$ & $0.866(0.078)$ & $0.932(0.039)$ \\
\hline
\end{tabular}

TABLE 1. Means and standard deviations (in brackets) of $\rho$ in the simulation study, scenario (T1). 

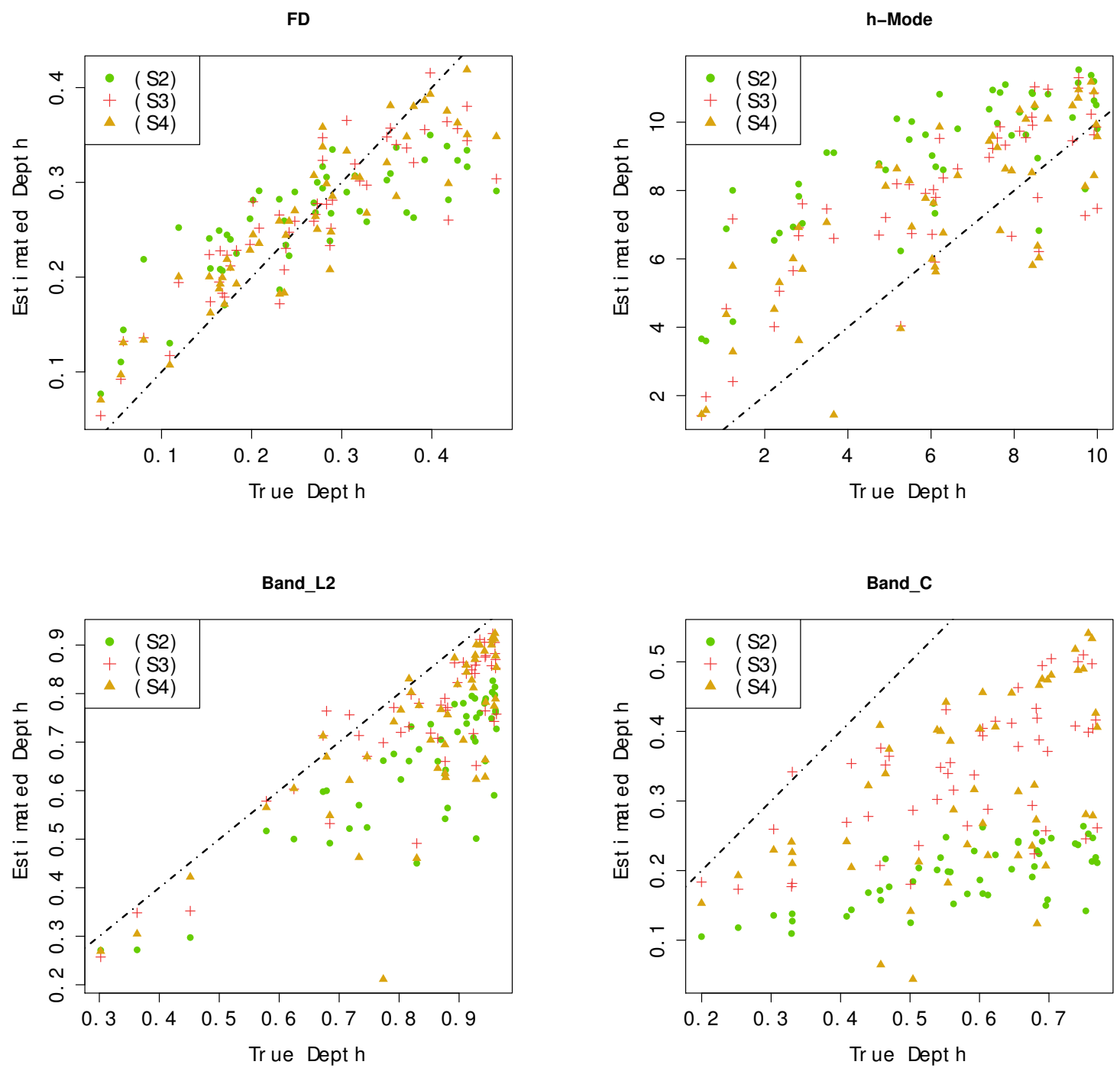

Figure 2. Plots of the true depth values $D\left(X_{i} ; P_{n}\right)$ against some of the estimated depths $D\left(\widetilde{X}_{i} ; \widetilde{P}_{n}\right)$ for one run of the simulation study, scenario (T2), (V2). The dashed-dotted line represents the axis of the first quadrant in $\mathbb{R}^{2}$. For curves reconstructed by (S2) the depth $h D_{1}$ tends to be biased upwards, while the bD depths are underestimated; for (S3) and (S4), no systematic bias is observed for depths based on the $L^{2}([0,1])$ norm.

To measure the approximation error, we use the Pearson correlation coefficient $\rho$ between $\left(D\left(X_{1}, P_{n}\right), \ldots, D\left(X_{n}, P_{n}\right)\right)$ and $\left(D\left(\widetilde{X}_{1}, \widetilde{P}_{n}\right), \ldots, D\left(\widetilde{X}_{n}, \widetilde{P}_{n}\right)\right)$. Other association/loss measures (such as the Spearman correlation, or MSE) provide the same conclusions, see the extended simulation study in the Supplementary Material.

In Tables 1-3 we see the results of the simulation study for scenarios (T1)-(T3), respectively. The reported values are the mean and the standard deviation (in brackets) of 


\begin{tabular}{|c|c|c|c|c|c|c|}
\hline & & (S1) & $(\mathrm{S} 2)$ & (S3) & (S4) & (S5) \\
\hline \multirow{4}{*}{ (V1) } & $a D$ & $0.717(0.074)$ & $0.863(0.036)$ & $0.916(0.024)$ & $0.924(0.021)$ & $0.920(0.022)$ \\
\hline & $h D_{1}$ & $0.700(0.082)$ & $0.809(0.060)$ & $0.829(0.054)$ & $0.768(0.092)$ & $0.807(0.074)$ \\
\hline & $h D_{2}$ & $0.623(0.094)$ & $0.655(0.093)$ & $0.580(0.107)$ & $0.400(0.138)$ & $0.458(0.130)$ \\
\hline & $b D_{1}$ & $0.852(0.081)$ & $0.881(0.058)$ & $0.891(0.051)$ & $0.791(0.116)$ & $0.849(0.085)$ \\
\hline & $b D_{2}$ & $0.724(0.079)$ & $0.778(0.067)$ & 0.691 & $0.508(0.118)$ & $0.557(0.109)$ \\
\hline & $\overline{a D}$ & $\overline{0} \overline{7} \overline{0} \overline{8}(0 . \overline{0} \overline{0})$ & $0 . \overline{804}(0.054)$ & $\overline{0} . \overline{8} \overline{6}(0.031)$ & $0 . \overline{8} 88(0.033)$ & $0.8 \overline{8} \overline{(0.035)}$ \\
\hline \multirow{3}{*}{$(\mathrm{V} 2)$} & $h D_{1}$ & $0.693(0.088)$ & $0.741(0.084)$ & $0.777(0.074)$ & $0.689(0.109)$ & $0.732(0.097)$ \\
\hline & $h D_{2}$ & $0.617(0.100)$ & $0.566(0.119)$ & $0.507(0.119)$ & $0.347(0.128)$ & $0.400(0.132)$ \\
\hline & $b D_{1}$ & $0.842(0.071)$ & $0.825(0.072)$ & $0.846(0.070)$ & $0.717(0.1$ & $0.770(0.116)$ \\
\hline \multirow{6}{*}{ (V3) } & $b D_{2}$ & $0.711(0.084)$ & $0.670(0.096)$ & $0.618(0.099)$ & $0.464(0.114)$ & $0.508(0.113)$ \\
\hline & $a \bar{D}$ & $\overline{0} \overline{7} \overline{2} \overline{7}(0.075)$ & $0 . \overline{4} 6(0.020)$ & $0.9 \overline{6} 0(0.013)$ & $0 . \overline{9} 71(0.010)$ & $0.96 \overline{8}(0.012)$ \\
\hline & $h D_{1}$ & $0.711(0.078)$ & $0.857(0.050)$ & $0.869(0.049)$ & $0.890(0.063)$ & $0.913(0.056)$ \\
\hline & $h D_{2}$ & $0.638(0.092)$ & $0.604(0.098)$ & $0.597(0.109)$ & $0.644(0.122)$ & $0.713(0.112)$ \\
\hline & $b D_{1}$ & $0.853(0.055)$ & $0.916(0.048)$ & $0.922(0.047)$ & $0.888(0.107)$ & $0.920(0.084)$ \\
\hline & $b D_{2}$ & $0.725(0.073)$ & $0.644(0.090)$ & $0.710(0.087)$ & $0.676(0.114)$ & $0.740(0.108)$ \\
\hline
\end{tabular}

TABLE 2. Means and standard deviations (in brackets) of $\rho$ in the simulation study, scenario (T2).

\begin{tabular}{|c|c|c|c|c|c|c|}
\hline & & (S1) & $(\mathrm{S} 2)$ & (S3) & (S4) & (S5) \\
\hline \multirow{4}{*}{ (V1) } & $a D$ & $0.579(0.087)$ & $0.885(0.030)$ & $0.922(0.024)$ & $0.933(0.022)$ & $0.927(0.025)$ \\
\hline & $h D_{1}$ & $0.569(0.109)$ & $0.894(0.032)$ & $0.912(0.031)$ & $0.934(0.031)$ & $0.937(0.025)$ \\
\hline & $h D_{2}$ & $0.507(0.118)$ & $0.781(0.068)$ & $0.725(0.078)$ & $0.786(0.081)$ & $0.818(0.069)$ \\
\hline & $b D_{1}$ & $0.734(0.104)$ & $0.957(0.025)$ & $0.959(0.025)$ & $0.967(0.033)$ & $0.968(0.024)$ \\
\hline \multirow{5}{*}{$(\mathrm{V} 2)$} & $b D_{2}$ & $0.609(0.107)$ & $0.846(0.056)$ & $0.796(0.064)$ & $0.831(0.077)$ & $0.861 \quad(0.063)$ \\
\hline & $a D$ & $\overline{0} . \overline{5} \overline{7} 3(0.0 \overline{9})$ & $0 . \overline{83}(0.051)$ & $\overline{0} \overline{8} \overline{9} \overline{2}(0.0 \overline{3} 9)$ & $\overline{0} . \overline{9} 0 \overline{1}(\overline{0} . \overline{0} \overline{4})$ & $\overline{0.8 \overline{8}} \overline{(0.039)}$ \\
\hline & $h D_{1}$ & $0.553(0.111)$ & $0.823(0.065)$ & $0.850(0.059)$ & $0.862(0.054)$ & $0.869(0.051)$ \\
\hline & $h D_{2}$ & $0.487(0.118)$ & $0.596(0.123)$ & $0.595(0.111)$ & $0.594(0.112)$ & $0.655(0.098)$ \\
\hline & $b D_{1}$ & $0.717(0.117)$ & $0.903(0.054)$ & $0.914(0.051)$ & $0.912(0.062)$ & $0.919(0.051)$ \\
\hline \multirow{6}{*}{ (V3) } & $b D_{2}$ & $0.585(0.110)$ & $0.686(0.097)$ & $0.686(0.095)$ & $0.676(0.100)$ & $0.731(0.090)$ \\
\hline & $\overline{a D}$ & $\overline{0} . \overline{5} \overline{6}(0.114)$ & $0 . \overline{959}(0.014)$ & $\overline{0.9 \overline{6}} \overline{(0.013)}$ & $0 . \overline{9} 74(0.010)$ & $0.9 \overline{0} \overline{0}(0.011)$ \\
\hline & $h D_{1}$ & $0.564(0.128)$ & $0.925(0.030)$ & $0.941(0.027)$ & $0.959(0.035)$ & $0.972(0.019)$ \\
\hline & $h D_{2}$ & $0.500(0.135)$ & $0.683(0.093)$ & $0.726(0.079)$ & $0.829(0.089)$ & $0.909(0.054)$ \\
\hline & $b D_{1}$ & $0.729(0.120)$ & $0.970(0.024)$ & $0.975(0.021)$ & $0.970(0.048)$ & $0.983(0.026)$ \\
\hline & $b D_{2}$ & $0.600(0.121)$ & $0.683(0.084)$ & $0.796(0.066)$ & $0.850(0.092)$ & $0.927(0.057)$ \\
\hline
\end{tabular}

TABLE 3. Means and standard deviations (in brackets) of $\rho$ in the simulation study, scenario (T3).

$\rho$, computed from 100 independent runs. In each setup we clearly see that the naive constant interpolation (S1) provides rather faltering results. While linear interpolation (S2) improves on these, kernel and local polynomial estimators (S3), (S4) and (S5) certainly 
outperform their competitors in most considered scenarios, for all depth functions. Notable exceptions are scenarios (T2), (V1) and (V2), where especially the local polynomial estimators (S4) and (S5) perform rather poorly for the depths based on the supremum norm (9). This is explained by the fact that the local polynomial estimators are known to break down easily in the extrapolation task. If no points are observed near the boundary of $[0,1]$, the extrapolants (S4) and (S5) lose their stability, which affects the supremum distance between the curves profoundly.

In most of the scenarios, however, the reconstruction methods (S3)-(S5) perform well. Moreover, the variability of the results typically decreases for (S3)-(S5). This is confirmed also by Figure 2, and by additional simulations given in the Supplementary Material.

To illustrate the "dimensionality blessing" effect mentioned in Section 4.2, we perform another, short simulation study. Here, under the settings as above with (T1) and (V1) we compare three choices for the number of time points

(M1) $m_{i}=\lceil 20+i / 5\rceil$ for $i=1, \ldots, n$;

(M2) $m_{i}=\lceil 50+i / 5\rceil$ for $i=1, \ldots, n$; and

(M3) $m_{i}=\lceil 100+i / 5\rceil$ for $i=1, \ldots, n$.

Scenario (M2) was considered previously. As confirmed in Table 4 (and the corresponding part of Table 1 for scenario (M2)), the more high-dimensional the discrete observations are, the more accurately the functional data depth can be observed.

\begin{tabular}{|c|c|c|c|c|c|c|}
\hline & & (S1) & $(\mathrm{S} 2)$ & (S3) & $(\mathrm{S} 4)$ & (S5) \\
\hline \multirow{4}{*}{ (M1) } & $a D$ & $0.466(0.120)$ & $0.832(0.051)$ & $0.850(0.046)$ & $0.871(0.042)$ & $0.858(0.049)$ \\
\hline & $h D_{1}$ & $0.469(0.124)$ & $0.789(0.079)$ & $0.768(0.083)$ & $0.799(0.080)$ & $0.813(0.074)$ \\
\hline & $h D_{2}$ & $0.420(0.130)$ & $0.588(0.122)$ & $0.526(0.124)$ & $0.538(0.126)$ & $0.590(0.109)$ \\
\hline & $b D_{1}$ & $0.624(0.144)$ & $0.853(0.085)$ & $0.839(0.085)$ & $0.827(0.115)$ & 0.859 (0.098) \\
\hline \multirow{6}{*}{ (M3) } & $b D_{2}$ & $0.515(0.132)$ & $0.701(0.096)$ & $0.622(0.108)$ & $0.606(0.115)$ & $0.654(0.104)$ \\
\hline & $\bar{a} \bar{D}$ & $\overline{0.6 \overline{8}} \overline{3}(0.0 \overline{8} 5 \overline{)}$ & $0 . \overline{9} 13(\overline{0} . \overline{0} \overline{0})$ & $0.9 \overline{6} \overline{1}(0.012)$ & $\overline{0} . \overline{9} \overline{6} 6(0.01 \overline{1})$ & $0 . \overline{9} \overline{2} \overline{2}(0.013)$ \\
\hline & $h D_{1}$ & $0.661(0.090)$ & $0.929(0.022)$ & $0.967(0.013)$ & $0.974(0.012)$ & $0.972(0.011)$ \\
\hline & $h D_{2}$ & $0.587(0.100)$ & $0.854(0.043)$ & $0.867(0.050)$ & $0.863(0.063)$ & $0.876(0.054)$ \\
\hline & $b D_{1}$ & $0.829(0.071)$ & $0.981(0.009)$ & $0.987(0.008)$ & $0.988(0.008)$ & $0.987(0.006)$ \\
\hline & $b D_{2}$ & $0.691(0.083)$ & $0.902(0.036)$ & $0.909(0.041)$ & $0.898(0.055)$ & $0.911(0.046)$ \\
\hline
\end{tabular}

TABLE 4. Means and standard deviations (in brackets) of $\rho$ in the simulation study, scenarios (M1) and (M3). Corresponding results in scenario (M2) can be found in Table 1, part (V1).

Altogether, we may conclude that in accordance with the theoretical developments obtained in Sections 2-4, pre-smoothing of discretely observed noisy random functions is a reasonable method of preprocessing in order to obtain information about functional depth. Moreover, depths defined using the $L^{2}([0,1])$ norm $(1)$ are generally more computationally stable than the corresponding depths defined via the supremum norm (9).

Acknowledgement. The authors thank the Managing Editor, the Editor and two referees for their valuable comments. This research was carried out during the first author's visit at the University of Toulouse, supported by the Research Foundation - Flanders. The work is supported by the IAP research network no. P7/06 of the Federal Science Policy (Belgium). The first author gratefully acknowledges support from the Research 
Foundation - Flanders, the grant 18-00522Y of the Czech Science Foundation, and the PRIMUS/17/SCI/3 project of Charles University.

\section{REFERENCES}

Agostinelli, C. and Romanazzi, M. (2011). Local depth. J. Stat. Plan. Inference, 141(2):817-830.

Apostol, T. M. (1976). Introduction to analytic number theory. Springer-Verlag, New York-Heidelberg. Undergraduate Texts in Mathematics.

Billingsley, P. (1999). Convergence of probability measures. Wiley Series in Probability and Statistics: Probability and Statistics. John Wiley \& Sons Inc., New York, second edition. A Wiley-Interscience Publication.

Bogachev, V. I. (2007). Measure theory. Vol. II. Springer-Verlag, Berlin.

Bott, A.-K., Devroye, L., and Kohler, M. (2013). Estimation of a distribution from data with small measurement errors. Electron. J. Stat., 7:2457-2476.

Cabrera, J. L. O. (2012). locpol: Kernel local polynomial regression. R package version $0.6-0$.

Chakraborty, A. and Chaudhuri, P. (2014). The spatial distribution in infinite dimensional spaces and related quantiles and depths. Ann. Statist., 42(3):1203-1231.

Claeskens, G., Hubert, M., Slaets, L., and Vakili, K. (2014). Multivariate functional halfspace depth. J. Amer. Statist. Assoc., 109(505):411-423.

Crambes, C., Kneip, A., and Sarda, P. (2009). Smoothing splines estimators for functional linear regression. Ann. Statist., 37(1):35-72.

Cuevas, A. (2014). A partial overview of the theory of statistics with functional data. $J$. Stat. Plan. Inference, 147:1-23.

Cuevas, A., Febrero, M., and Fraiman, R. (2006). On the use of the bootstrap for estimating functions with functional data. Comput. Statist. Data Anal., 51(2):10631074.

Cuevas, A., Febrero, M., and Fraiman, R. (2007). Robust estimation and classification for functional data via projection-based depth notions. Comput. Statist., 22(3):481-496.

Cuevas, A. and Fraiman, R. (2009). On depth measures and dual statistics. A methodology for dealing with general data. J. Multivariate Anal., 100(4):753-766.

De Brabanter, K., Cao, F., Gijbels, I., and Opsomer, J. (2018). Local polynomial regression with correlated errors in random design and unknown correlation structure. Biometrika, 105(3):681-690.

Dudley, R. M. (1999). Uniform central limit theorems, volume 63 of Cambridge Studies in Advanced Mathematics. Cambridge University Press, Cambridge.

Dudley, R. M. (2002). Real analysis and probability, volume 74 of Cambridge Studies in Advanced Mathematics. Cambridge University Press, Cambridge. Revised reprint of the 1989 original.

Dutta, S., Ghosh, A. K., and Chaudhuri, P. (2011). Some intriguing properties of Tukey's half-space depth. Bernoulli, 17(4):1420-1434.

Eichelsbacher, P. and Schmock, U. (2003). Rank-dependent moderate deviations of $U$ empirical measures in strong topologies. Probab. Theory Related Fields, 126(1):61-90.

Fan, J. and Gijbels, I. (1996). Local polynomial modelling and its applications, volume 66 of Monographs on Statistics and Applied Probability. Chapman \& Hall, London.

Ferraty, F. and Vieu, P. (2006). Nonparametric functional data analysis. Theory and practice. Springer Series in Statistics. Springer, New York. 
Fraiman, R. and Muniz, G. (2001). Trimmed means for functional data. Test, 10(2):419440.

Genton, M. G., Johnson, C., Potter, K., Stenchikov, G., and Sun, Y. (2014). Surface boxplots. Stat, 3(1):1-11.

Gijbels, I. and Nagy, S. (2015). Consistency of non-integrated depths for functional data. J. Multivariate Anal., 140:259 - 282.

Goia, A. and Vieu, P. (2016). An introduction to recent advances in high/infinite dimensional statistics [Editorial]. J. Multivariate Anal., 146:1-6.

Grinblat, L. Š. (1976). A limit theorem for measurable random processes and its applications. Proc. Amer. Math. Soc., 61(2):371-376 (1977).

Györfi, L., Kohler, M., Krzyżak, A., and Walk, H. (2002). A distribution-free theory of nonparametric regression. Springer Series in Statistics. Springer-Verlag, New York.

Horváth, L. and Kokoszka, P. (2012). Inference for functional data with applications. Springer Series in Statistics. Springer, New York.

Hsing, T. and Eubank, R. (2015). Theoretical foundations of functional data analysis, with an introduction to linear operators. Wiley Series in Probability and Statistics. John Wiley \& Sons, Ltd., Chichester.

Hyndman, R. J. and Shang, H. L. (2010). Rainbow plots, bagplots, and boxplots for functional data. J. Comput. Graph. Statist., 19(1):29-45.

Ieva, F. and Paganoni, A. M. (2013). Depth measures for multivariate functional data. Comm. Statist. Theory Methods, 42(7):1265-1276.

Ivanov, A. V. (1980). Convergence of distributions of functionals of measurable random fields. Ukrain. Mat. Zh., 32(1):27-34, 141.

Jiang, C.-R. and Wang, J.-L. (2010). Covariate adjusted functional principal components analysis for longitudinal data. Ann. Statist., 38(2):1194-1226.

Kohler, M., Krzyżak, A., and Walk, H. (2009). Optimal global rates of convergence for nonparametric regression with unbounded data. J. Stat. Plan. Inference, 139(4):12861296.

Li, Y. and Hsing, T. (2010). Uniform convergence rates for nonparametric regression and principal component analysis in functional/longitudinal data. Ann. Statist., 38(6):3321-3351.

Liu, R. Y. (1990). On a notion of data depth based on random simplices. Ann. Statist., 18(1):405-414.

Liu, X.-H. (2001). Kernel smoothing for spatially correlated data. Ph.D. thesis, Department of Statistics, Iowa State University.

López-Pintado, S. and Jornsten, R. (2007). Functional analysis via extensions of the band depth. In Complex datasets and inverse problems, volume 54 of IMS Lecture Notes Monogr. Ser., pages 103-120. Inst. Math. Statist., Beachwood, OH.

López-Pintado, S. and Romo, J. (2009). On the concept of depth for functional data. J. Amer. Statist. Assoc., 104(486):718-734.

López-Pintado, S. and Romo, J. (2011). A half-region depth for functional data. Comput. Statist. Data Anal., 55(4):1679-1695.

López-Pintado, S., Sun, Y., Lin, J., and Genton, M. (2014). Simplicial band depth for multivariate functional data. Adv. Data Anal. Classif., 8(3):321-338.

López-Pintado, S. and Wei, Y. (2011). Depth for sparse functional data. In Recent advances in functional data analysis and related topics, Contrib. Statist., pages 209212. Physica-Verlag/Springer, Heidelberg. 
Mosler, K. (2013). Depth statistics. In Becker, C., Fried, R., and Kuhnt, S., editors, Robustness and complex data structures, pages 17-34. Springer, Heidelberg.

Mosler, K. and Polyakova, Y. (2012). General notions of depth for functional data. arXiv preprint arXiv:1208.1981.

Müller, H.-G., Wu, Y., and Yao, F. (2013). Continuously additive models for nonlinear functional regression. Biometrika, 100(3):607-622.

Nagy, S. (2015). Consistency of h-mode depth. J. Stat. Plan. Inference, 165:91-103.

Nagy, S. (2017a). Integrated depth for measurable functions and sets. Statist. Probab. Lett., 123:165-170.

Nagy, S. (2017b). An overview of consistency results for depth functionals. In Aneiros, G., Bongiorno, E. G., Cao, R., and Vieu, P., editors, Functional Statistics and Related Fields, pages 189-196. Springer International Publishing, Cham.

Nagy, S., Gijbels, I., and Hlubinka, D. (2016a). Weak convergence of discretely observed functional data with applications. J. Multivariate Anal., 146:46 - 62. Special Issue on Statistical Models and Methods for High or Infinite Dimensional Spaces.

Nagy, S., Gijbels, I., Omelka, M., and Hlubinka, D. (2016b). Integrated depth for functional data: statistical properties and consistency. ESAIM Probab. Stat., 20:95-130.

Narisetty, N. N. and Nair, V. N. (2016). Extremal depth for functional data and applications. J. Amer. Statist. Assoc., 111(516):1705-1714.

Opsomer, J., Wang, Y., and Yang, Y. (2001). Nonparametric regression with correlated errors. Statist. Sci., 16(2):134-153.

Paul, D. and Peng, J. (2009). Consistency of restricted maximum likelihood estimators of principal components. Ann. Statist., 37(3):1229-1271.

Pokotylo, O., Mozharovskyi, P., Dyckerhoff, R., and Nagy, S. (2017). ddalpha: DepthBased Classification and Calculation of Data Depth. R package version 1.3.1.

R Core Team (2015). R: A Language and Environment for Statistical Computing. R Foundation for Statistical Computing, Vienna, Austria.

Radchenko, P., Qiao, X., and James, G. M. (2015). Index models for sparsely sampled functional data. J. Amer. Statist. Assoc., 110(510):824-836.

Ramsay, J. O. and Silverman, B. W. (2005). Functional data analysis. Springer Series in Statistics. Springer, New York, Second edition.

Stone, C. J. (1977). Consistent nonparametric regression. Ann. Statist., 5(4):595-645.

Stone, C. J. (1982). Optimal global rates of convergence for nonparametric regression. Ann. Statist., 10(4):1040-1053.

Sun, Y. and Genton, M. G. (2011). Functional boxplots. J. Comput. Graph. Statist., 20(2):316-334.

Sun, Y. and Genton, M. G. (2012). Adjusted functional boxplots for spatio-temporal data visualization and outlier detection. Environmetrics, 23(1):54-64.

Tukey, J. W. (1975). Mathematics and the picturing of data. In Proceedings of the International Congress of Mathematicians (Vancouver, B. C., 1974), Vol. 2, pages 523-531. Canad. Math. Congress, Montreal, Que.

Varadarajan, V. S. (1958). On the convergence of sample probability distributions. Sankhyā, 19:23-26.

Whitaker, R. T., Mirzargar, M., and Kirby, R. M. (2013). Contour boxplots: A method for characterizing uncertainty in feature sets from simulation ensembles. IEEE Trans. Vis. Comput. Graph., 19(12):2713-2722. 
Wu, Y., Fan, J., and Müller, H.-G. (2010). Varying-coefficient functional linear regression. Bernoulli, 16(3):730-758.

Yao, F., Müller, H.-G., and Wang, J.-L. (2005). Functional data analysis for sparse longitudinal data. J. Amer. Statist. Assoc., 100(470):577-590.

Zhang, X. and Wang, J.-L. (2016). From sparse to dense functional data and beyond. Ann. Statist., 44(5):2281-2321.

Zuo, Y. and Serfling, R. (2000). General notions of statistical depth function. Ann. Statist., 28(2):461-482. 


\section{Appendix A. Proofs of the Theoretical Results}

A.1. Proof of Theorem 1. Without loss of generality, assume that in (A2) $B \leq 1$, otherwise continue with the kernel $K / B$. Using the independence of the random function $X$, observation points $T$ and the noise $\varepsilon$ we can write

$$
\begin{aligned}
c_{T} \mathrm{E}\left\|\tilde{X}_{n}-X_{n}\right\|^{2} & =\mathrm{E} \int_{0}^{1}\left(\tilde{X}_{n}(t)-X_{n}(t)\right)^{2} c_{T} \mathrm{~d} \lambda(t) \\
& \leq \mathrm{E} \int_{0}^{1}\left(\tilde{X}_{n}(t)-X_{n}(t)\right)^{2} \mathrm{~d} P_{T}(t) .
\end{aligned}
$$

Let us first show that the last term above vanishes with $n \rightarrow \infty$.

For a fixed $x \in L^{2}([0,1])$ observed within the design

$$
x_{j}^{*}=x\left(T_{j}\right)+\varepsilon_{j} \quad \text { for } j=1, \ldots, m
$$

we can write for $w_{j}(t)=K\left(\frac{t-T_{j}}{h_{m}}\right) / \sum_{k=1}^{m} K\left(\frac{t-T_{k}}{h_{m}}\right)$ the following

$$
\begin{aligned}
\mathrm{E}_{\varepsilon, T} \int_{0}^{1}(\widetilde{x}(t)-x(t))^{2} \mathrm{~d} P_{T}(t) & \leq 2 \mathrm{E}_{\varepsilon, T} \int_{0}^{1} \widetilde{x}(t)^{2} \mathrm{~d} P_{T}(t)+2 \mathrm{E}_{\varepsilon, T} \int_{0}^{1} x(t)^{2} \mathrm{~d} P_{T}(t) \\
& =2 \mathrm{E}_{\varepsilon, T} \int_{0}^{1}\left(\sum_{j=1}^{m} w_{j}(t) x_{j}^{*}\right)^{2} \mathrm{~d} P_{T}(t)+2 \mathrm{E}_{T} x(T)^{2} \\
& \leq 2 \mathrm{E}_{\varepsilon, T} \int_{0}^{1} \sum_{j=1}^{m} w_{j}(t)\left(x_{j}^{*}\right)^{2} \mathrm{~d} P_{T}(t)+2 \mathrm{E}_{T} x(T)^{2} \\
& =2 \int_{0}^{1} \sum_{j=1}^{m} \mathrm{E}_{\varepsilon, T} w_{j}(t)\left(x\left(T_{j}\right)+\varepsilon_{j}\right)^{2} \mathrm{~d} P_{T}(t)+2 \mathrm{E}_{T} x(T)^{2} \\
& \leq 4 \int_{0}^{1} \sum_{j=1}^{m} \mathrm{E}_{\varepsilon, T} w_{j}(t)\left(x\left(T_{j}\right)^{2}+\varepsilon_{j}^{2}\right) \mathrm{d} P_{T}(t)+2 \mathrm{E}_{T} x(T)^{2} \\
& \leq 4\left(\sigma^{2}+\int_{0}^{1} \sum_{j=1}^{m} \mathrm{E}_{T} w_{j}(t) x\left(T_{j}\right)^{2} \mathrm{~d} P_{T}(t)\right)+2 \mathrm{E}_{T} x(T)^{2} .
\end{aligned}
$$

Now, in the first expression on the right hand side we have the kernel estimate for the function $x^{2}$ based on the observations $x^{2}\left(T_{j}\right)$ for $j=1, \ldots, m$ (without noise, that is $\sigma^{2}(t)=0$ for all $t \in[0,1]$ ). For this term, it is known (see Györfi et al., 2002, proof of Theorem 5.1) that for some $C>0$ for any $x \in L^{2}([0,1])$ such that

$$
\mathrm{E}_{T} x(T)^{2}<\infty
$$

and for any $m \geq 1$ we can bound

$$
\int_{0}^{1} \sum_{j=1}^{m} \mathrm{E}_{T} w_{j}(t) x\left(T_{j}\right)^{2} \mathrm{~d} P_{T}(t) \leq C \mathrm{E}_{T} x(T)^{2},
$$

meaning that we can continue the chain of inequalities above by writing

$$
\mathrm{E}_{\varepsilon, T} \int_{0}^{1}(\widetilde{x}(t)-x(t))^{2} \mathrm{~d} P_{T}(t) \leq 4 \sigma^{2}+(4 C+2) \mathrm{E}_{T} x(T)^{2} .
$$


Since (18) is valid for any $x \in L^{2}([0,1])$ such that $(17)$ is true, we may as well replace the fixed function $x$ by the random function $X \sim P$, apply expectation with respect to $X$ to both sides of (18), and get

$$
\mathrm{E} \int_{0}^{1}(\widetilde{X}(t)-X(t))^{2} \mathrm{~d} P_{T}(t) \leq 4 \sigma^{2}+(4 C+2) \mathrm{E} X(T)^{2},
$$

where the right hand side is finite by (A1) and (A4). Note that (17) is necessarily true for P-almost all realisations of $X$ by (A1).

By Stone (1977, Theorem 1) using (A2)-(A5) for any fixed $x_{n} \in L^{2}([0,1])$ (here, the subscript $n$ stands for the fact that $x$ is assumed to be observed at $\left(T_{n, 1}, \ldots, T_{n, m_{n}}\right)$ with the additive noise $\left.\left(\varepsilon_{n, 1}, \ldots, \varepsilon_{n, m_{n}}\right)\right)$

$$
\mathrm{E}_{\varepsilon, T} \int_{0}^{1}\left(\widetilde{x}_{n}(t)-x_{n}(t)\right)^{2} \mathrm{~d} P_{T}(t) \underset{n \rightarrow \infty}{\longrightarrow} 0,
$$

see also Györfi et al. (2002, Theorem 4.1 and Theorem 5.1). To allow for the replacement of $x$ by the random $X$ as above, it is enough to apply the expectation with respect to $X$ to this formula. The dominated convergence theorem (see Dudley, 2002, Theorem 4.3.5) then asserts that

$$
\mathrm{E} \int_{0}^{1}\left(\widetilde{X}_{n}(t)-X_{n}(t)\right)^{2} \mathrm{~d} P_{T}(t) \underset{n \rightarrow \infty}{\longrightarrow} 0 .
$$

The boundedness assumption of the integrand function for the dominated convergence theorem was verified in (19).

The rest of the proof is straightforward. By (16) and the previous results

$$
\lim _{n \rightarrow \infty} \mathrm{E}\left\|\widetilde{X}_{n}-X_{n}\right\|^{2}=0
$$

Consequently, by the Chebyshev inequality (see Dudley, 2002, 8.3.1) for any $\varepsilon>0$

$$
\lim _{n \rightarrow \infty} \mathrm{P}\left(\left\|\tilde{X}_{n}-X_{n}\right\|>\varepsilon\right)=0,
$$

and by Dudley (2002, Proposition 9.3.5) we can conclude that $\widetilde{X}_{n}$ converges in law to $X$. The assertion of Theorem 1 then follows by the same argument as Nagy et al. (2016a, Theorem 1, Part (ii)).

A.2. Proof of Theorem 2. The proof is based on the following lemma establishing the uniform qualitative robustness of all the concerned depths over the space $L^{2}([0,1])$.

Lemma 1. Let $P_{\nu} \underset{\nu \rightarrow \infty}{\stackrel{w}{\longrightarrow}} P$ in $\mathcal{P}\left(L^{2}([0,1])\right)$. Then

$$
\sup _{x \in L^{2}([0,1])}\left|D\left(x ; P_{\nu}\right)-D(x ; P)\right| \underset{\nu \rightarrow \infty}{\longrightarrow} 0,
$$

if $D$ is $h D$ or $b D$. Moreover, if also (A6) is true for $P$, then the result holds true also for $D=a D$.

Proof. As shown in Nagy (2015, proof of Theorem 1)

$$
\left\{x_{1} \mapsto \frac{1}{h(P)} K_{D}\left(\frac{\left\|x-x_{1}\right\|}{h(P)}\right): x \in L^{2}([0,1])\right\}
$$

is a set of uniformly equicontinuous, and uniformly bounded functionals $L^{2}([0,1]) \rightarrow \mathbb{R}$. Therefore, for $h D$, the result follows from Dudley (2002, Corollary 11.3.4). 
For $b D$, we show a similar continuity result for the set

$$
\left\{\left(x_{1}, \ldots, x_{J}\right) \mapsto K_{D}\left(d\left(x, B\left(x_{1}, \ldots, x_{J}\right)\right)\right): x \in L^{2}([0,1])\right\} .
$$

First of all, notice that $K_{D}$ is uniformly continuous and thus its minimal modulus of continuity

$$
\delta_{K_{D}}(\alpha)=\sup _{|s-t| \leq \alpha}\left|K_{D}(s)-K_{D}(t)\right|
$$

vanishes as $\alpha \rightarrow 0$. From the definition of $\delta_{K_{D}}$ we have

$$
\begin{aligned}
& \left|K_{D}\left(d\left(x, B\left(x_{1}, \ldots, x_{J}\right)\right)\right)-K_{D}\left(d\left(x, B\left(y_{1}, \ldots, y_{J}\right)\right)\right)\right| \\
& \quad \leq \delta_{K_{D}}\left(d\left(x, B\left(x_{1}, \ldots, x_{J}\right)\right)-d\left(x, B\left(y_{1}, \ldots, y_{J}\right)\right)\right) .
\end{aligned}
$$

Assume now that for $\varepsilon>0$ it is true that

$$
\left\|x_{j}-y_{j}\right\| \leq \varepsilon \text { for all } j=1, \ldots, J .
$$

Denote by $m \in B\left(x_{1}, \ldots, x_{J}\right)$ the function for which $d\left(x, B\left(x_{1}, \ldots, x_{J}\right)\right)=\|x-m\|$. Such a function exists because of the construction of the band of functions. In what follows we show that if $(22)$ is true, then

$$
d\left(m, B\left(y_{1}, \ldots, y_{J}\right)\right) \leq 4 J \varepsilon,
$$

meaning that

$$
d\left(x, B\left(y_{1}, \ldots, y_{J}\right)\right) \leq\|x-m\|+d\left(m, B\left(y_{1}, \ldots, y_{J}\right)\right) \leq d\left(x, B\left(x_{1}, \ldots, x_{J}\right)\right)+4 J \varepsilon .
$$

Because in the beginning the role of $B\left(x_{1}, \ldots, x_{J}\right)$ and $B\left(y_{1}, \ldots, y_{J}\right)$ is symmetric, the last expression implies that if (22) is in order, then the right hand side in (21) can be bounded from above by $\delta_{K_{D}}(4 J \varepsilon)$. Because the modulus $\delta_{K_{D}}(\alpha)$ vanishes as $\alpha \rightarrow 0$, the uniform continuity of (20) follows.

To show (23), consider first $l_{x} \in L^{2}([0,1])$ defined by $l_{x}(t)=\min _{j=1, \ldots, J} x_{j}(t)$ for $t \in[0,1]$, and $l_{y} \in L^{2}([0,1])$ given analogously for functions $y_{j}$. Partition $[0,1]$ into two disjoint subsets $I_{x}$ and $I_{y}$ given by

$$
\begin{aligned}
& I_{x}=\left\{t \in[0,1]: l_{x}(t) \leq l_{y}(t)\right\}, \\
& I_{y}=\left\{t \in[0,1]: l_{y}(t)<l_{x}(t)\right\} .
\end{aligned}
$$

Then, partition the set $I_{x}$ further into $I_{x_{j}}=\left\{t \in[0,1]: x_{j}(t)=l_{x}(t)\right\}$ and the set $I_{y}$ accordingly. Assume that the sets $I_{x_{1}}, \ldots, I_{x_{J}}, I_{y_{1}}, \ldots, I_{y_{J}}$ do not overlap; if they do, modify them by assigning the points in the intersections to one of the intersecting sets so that the new partition consists of pairwise disjoint measurable sets whose union is $[0,1]$. This is always possible, since we deal with a finite number of measurable functions.

Writing $\mathbb{I}\left[I_{x}\right]$ for $\mathbb{I}\left[t \in I_{x}\right]$ etc., we can use the construction of the partition above and (22) and bound

$$
\left\|\left(l_{x}-l_{y}\right) \mathbb{I}\left[I_{x_{j}}\right]\right\|=\left\|\left(x_{j}-l_{y}\right) \mathbb{I}\left[I_{x_{j}}\right]\right\| \leq\left\|\left(x_{j}-y_{j}\right) \mathbb{I}\left[I_{x_{j}}\right]\right\| \leq\left\|x_{j}-y_{j}\right\| \leq \varepsilon
$$

leading to

$$
\begin{aligned}
\left\|l_{x}-l_{y}\right\| & =\left\|\left(l_{x}-l_{y}\right) \mathbb{I}\left[I_{x}\right]\right\|+\left\|\left(l_{x}-l_{y}\right) \mathbb{I}\left[I_{y}\right]\right\| \\
& =\sum_{j=1}^{J}\left\|\left(l_{x}-l_{y}\right) \mathbb{I}\left[I_{x_{j}}\right]\right\|+\sum_{j=1}^{J}\left\|\left(l_{x}-l_{y}\right) \mathbb{I}\left[I_{y_{j}}\right]\right\| \leq 2 J \varepsilon .
\end{aligned}
$$

Analogously, one can prove that also for functions $u_{x}(t)=\max _{j=1, \ldots, J} x_{j}(t)$ and $u_{y}(t)=$ $\max _{j=1, \ldots, J} y_{j}(t)$ it holds true that $\left\|u_{x}-u_{y}\right\| \leq 2 J \varepsilon$. 
Now, consider $m \in B\left(x_{1}, \ldots, x_{J}\right)$ and similarly as before, define three disjoint sets partitioning $[0,1]$

$$
\begin{aligned}
& I_{1}=\left\{t \in[0,1]: m(t)<l_{y}(t)\right\}, \\
& I_{2}=\left\{t \in[0,1]: l_{y}(t) \leq m(t) \leq u_{y}(t)\right\}, \\
& I_{3}=\left\{t \in[0,1]: u_{y}(t)<m(t)\right\} .
\end{aligned}
$$

With this notation, one can write for $m \in B\left(x_{1}, \ldots, x_{J}\right)$

$$
\begin{aligned}
d\left(m, B\left(y_{1}, \ldots, y_{J}\right)\right) & \leq\left\|\left(m-l_{y}\right) \mathbb{I}\left[I_{1}\right]\right\|+\left\|(m-m) \mathbb{I}\left[I_{2}\right]\right\|+\left\|\left(m-u_{y}\right) \mathbb{I}\left[I_{3}\right]\right\| \\
& \leq\left\|\left(l_{x}-l_{y}\right) \mathbb{I}\left[I_{1}\right]\right\|+\left\|\left(u_{x}-u_{y}\right) \mathbb{I}\left[I_{3}\right]\right\| \leq 4 J \varepsilon .
\end{aligned}
$$

Hence, by showing (23) the uniform continuity of (20) is verified.

To conclude the proof for $b D$, for any $P \in \mathcal{P}\left(L^{2}([0,1])\right)$ denote the $J$ th product measure of $P$ by $P^{J} \in \mathcal{P}\left(L^{2}([0,1])^{J}\right)$, where $L^{2}([0,1])^{J}$ is the $J$ th Cartesian product of the space $L^{2}([0,1])$. Now it is enough to use the result of Billingsley (1999, Theorem 2.8) which provides that if $P_{\nu} \underset{\nu \rightarrow \infty}{\stackrel{w}{\longrightarrow}} P$, then $P_{\nu}^{J} \underset{\nu \rightarrow \infty}{\stackrel{w}{\longrightarrow}} P^{J}$. The assertion of the theorem for $b D$ then follows from Dudley (2002, Corollary 11.3.4).

For the integrated depth one can modify the proof of Nagy et al. (2016b, Theorem 11) using the measurability result established in Nagy (2017a, Theorem 1) to obtain the result. Note that for the consistency of the integrated depth, the condition (A6) is not necessary.

Having established Lemma 1, the assertions for $h D$ and $a D$ follow directly by application of the Varadarajan theorem (see Dudley, 2002, Theorem 11.4.1).

For $b D$, the proof is slightly more complicated because the sample version of the depth takes the form of a U-statistic. For this, we need to introduce some additional notation. Recall that for $P \in \mathcal{P}\left(L^{2}([0,1])\right)$ and $J \geq 1, P^{J} \in \mathcal{P}\left(L^{2}([0,1])^{J}\right)$ stands for the $J$ th product measure of $P$. For $P_{n}=P_{n}(\omega) \in \mathcal{P}\left(L^{2}([0,1])\right)$ the empirical measure of $X_{1}, \ldots, X_{n} \in L^{2}([0,1])$, define the $J$ th U-empirical measure (see Eichelsbacher and Schmock, 2003) as

$$
L_{n}^{J}=\frac{1}{n_{(J)}} \sum_{i_{1}, \ldots, i_{J} \in I(J, n)} \delta_{\left(X_{i_{1}}, \ldots, X_{i_{J}}\right)} .
$$

Here, $n^{(J)}=\prod_{k=0}^{J-1}(n-k), I(J, n) \subset\{1, \ldots, n\}^{J}$ consists of all $J$-tuples with pairwise different components, and $\delta_{x}$ is the Dirac measure of $x \in L^{2}([0,1])^{J}$. It is possible to write

$$
\begin{aligned}
b D\left(x ; P_{n}\right) & =\int_{L^{2}([0,1])^{J}} K_{D}\left(d\left(x, B\left(y_{1}, \ldots, y_{J}\right)\right)\right) \mathrm{d} L_{n}^{J}\left(y_{1}, \ldots, y_{J}\right), \\
b D(x ; P) & =\int_{L^{2}([0,1])^{J}} K_{D}\left(d\left(x, B\left(y_{1}, \ldots, y_{J}\right)\right)\right) \mathrm{d} P^{J}\left(y_{1}, \ldots, y_{J}\right) .
\end{aligned}
$$

The measure $L_{n}^{J}$ can be also written as

$$
L_{n}^{J}=\frac{n^{J}}{n_{(J)}} P_{n}^{J}+\left(1-\frac{n^{J}}{n_{(J)}}\right) Q_{n}^{J}
$$

where $Q_{n}^{J}=Q_{n}^{J}(\omega) \in \mathcal{P}\left(L^{2}([0,1])^{J}\right)$ is the uniform probability measure over those $\left(X_{i_{1}}, \ldots, X_{i_{J}}\right) \in L^{2}([0,1])^{J}$ such that $i_{j}=i_{k}$ for some $j \neq k$. The next lemma will be used to assert the equivalence of the weak convergence of $L_{n}^{J}$ and $P_{n}^{J}$. 
Lemma 2. Let $P_{\nu}, Q_{\nu}, P \in \mathcal{P}\left(L^{2}([0,1])^{J}\right)$ and $\left\{\alpha_{\nu}\right\}_{\nu=1}^{\infty} \subset[0,1]$ be such that $\alpha_{\nu} \underset{\nu \rightarrow \infty}{\longrightarrow} 1$. Then $\alpha_{\nu} P_{\nu}+\left(1-\alpha_{\nu}\right) Q_{\nu} \underset{\nu \rightarrow \infty}{\stackrel{w}{\longrightarrow}} P$ if and only if $P_{\nu} \underset{\nu \rightarrow \infty}{\stackrel{w}{\longrightarrow}} P$.

Proof. For the proof, consider the Kantorovich-Rubinstein norm (Bogachev, 2007, Chapter 8.3) $\|\cdot\|_{0}$ generating the weak topology on the space of non-negative measures on $L^{2}([0,1])^{J}$. For the "if" part of the proof we can write

$$
\begin{aligned}
0 & \leq\left\|\alpha_{\nu} P_{\nu}+\left(1-\alpha_{\nu}\right) Q_{\nu}-P\right\|_{0} \leq \alpha_{\nu}\left\|P_{\nu}-P\right\|_{0}+\left(1-\alpha_{\nu}\right)\left\|Q_{\nu}-P\right\|_{0} \\
& \leq\left\|P_{\nu}-P\right\|_{0}+\left(1-\alpha_{\nu}\right) 2
\end{aligned}
$$

and as $\nu \rightarrow \infty$, the right hand side vanishes.

For the opposite implication we can find a similar bound

$$
\begin{aligned}
0 & \leq\left\|P_{\nu}-P\right\|_{0} \leq\left\|P_{\nu}-\alpha_{\nu} P_{\nu}-\left(1-\alpha_{\nu}\right) Q_{\nu}\right\|_{0}+\left\|\alpha_{\nu} P_{\nu}+\left(1-\alpha_{\nu}\right) Q_{\nu}-P\right\|_{0} \\
& \leq\left(1-\alpha_{\nu}\right) 2+\left\|\alpha_{\nu} P_{\nu}+\left(1-\alpha_{\nu}\right) Q_{\nu}-P\right\|_{0},
\end{aligned}
$$

yielding the result.

Applying Lemma 2 to the decomposition of $L_{n}^{J}$ from (24) asserts that for any $\omega \in \Omega$ fixed $L_{n}^{J}(\omega) \underset{n \rightarrow \infty}{\stackrel{w}{\longrightarrow}} P^{J}$ is equivalent with $P_{n}^{J}(\omega) \underset{n \rightarrow \infty}{\stackrel{w}{\longrightarrow}} P^{J}$. The latter is, by Varadarajan's theorem (Dudley, 2002, Theorem 11.4.1) and Billingsley (1999, Theorem 2.8), true for P-almost all $\omega \in \Omega$. Therefore, we obtain that

$$
\mathrm{P}\left(L_{n}^{J} \underset{n \rightarrow \infty}{\stackrel{w}{\longrightarrow}} P^{J}\right)=\mathrm{P}\left(P_{n}^{J} \underset{n \rightarrow \infty}{\stackrel{w}{\longrightarrow}} P^{J}\right)=1 .
$$

This formula together with Lemma 1 provides the assertion for $b D$.

A.3. Proof of Theorem 3. Apply Theorem 1 together with Lemma 1. For $b D$, modify the proof according to the proof of Theorem 2.

A.4. Proof of Theorem 4. First, notice that

$$
\begin{aligned}
\sup _{x \in L^{2}([0,1])}\left|a D\left(x ; P_{n}\right)-a D(x ; P)\right| & =\sup _{x \in L^{2}([0,1])}\left|\int_{0}^{1} D_{1}\left(x(t) ; P_{n, t}\right)-D_{1}\left(x(t) ; P_{t}\right) \mathrm{d} \lambda(t)\right| \\
& \leq \int_{0}^{1} \sup _{u \in \mathbb{R}}|| 1 / 2-F_{t}(u)|-| 1 / 2-F_{n, t}(u)|| \mathrm{d} \lambda(t) \\
& \leq \int_{0}^{1} \sup _{u \in \mathbb{R}}\left|F_{n, t}(u)-F_{t}(u)\right| \mathrm{d} \lambda(t) .
\end{aligned}
$$

By Lemma 3 given in Appendix A.7 and

$$
\mathrm{P}\left(\sup _{x \in L^{2}([0,1])}\left|a D\left(x ; P_{n}\right)-a D(x ; P)\right|>\frac{\varepsilon}{\sqrt{n}}\right) \leq \mathrm{P}\left(\int_{0}^{1} \xi_{n}(t) \mathrm{d} \lambda(t)>\varepsilon\right) \text { for all } n \geq 1,
$$

where $\xi_{n}$ is defined in (30), we obtain the desired result. 
A.5. Proof of Theorem 5. By a straightforward modification of Györfi et al. (2002, Theorem 5.2 and Problem 5.6), under the conditions assumed in Theorem 5, the rate of convergence as stated holds true for a single fixed function $x$. Notice that in the proof of Györfi et al. (2002, Theorem 5.2), by taking $\mathrm{E}_{X}$ on the both sides of the last chain of inequalities in the proof and using (16), we see that (in our notation, for $\beta=1$ and the naive kernel as considered in the proof presented in the book)

$$
\begin{aligned}
c_{T} \mathrm{E}\left\|X_{n}-\widetilde{X}_{n}\right\|^{2} \leq & 2 \sigma^{2} \frac{\tilde{c}}{m_{n} h_{m_{n}}}+L^{2} h_{m_{n}}^{2} \\
& +\mathrm{E}_{X} \int_{0}^{1} X(t)^{2}\left(1-P_{T}\left(\left[t-h_{m_{n}}, t+h_{m_{n}}\right]\right)\right)^{m_{n}} \mathrm{~d} P_{T}(t),
\end{aligned}
$$

for some constant $\tilde{c}>0$. Here, the last summand can be bounded from above using Fubini's theorem, $\left(\mathrm{A} 1^{*}\right)$ and (A3) by

$$
\begin{aligned}
\int_{0}^{1} \mathrm{E}_{X} X(t)^{2} & \left(1-P_{T}\left(\left[t-h_{m_{n}}, t+h_{m_{n}}\right]\right)\right)^{m_{n}} \mathrm{~d} P_{T}(t) \\
& \leq M \int_{0}^{1}\left(1-P_{T}\left(\left[t-h_{m_{n}}, t+h_{m_{n}}\right]\right)\right)^{m_{n}} \mathrm{~d} P_{T}(t) \\
& \leq M \int_{0}^{1} \exp \left(-m_{n} P_{T}\left(\left[t-h_{m_{n}}, t+h_{m_{n}}\right]\right)\right) \mathrm{d} P_{T}(t) \\
& \leq M \sup _{u>0}(u \exp (-u)) \int_{0}^{1} \frac{1}{m_{n} P_{T}\left(\left[t-h_{m_{n}}, t+h_{m_{n}}\right]\right)} \mathrm{d} P_{T}(t) \\
& \leq \frac{M}{\exp (1)} \frac{1}{2 m_{n} h_{m_{n}} c_{T}},
\end{aligned}
$$

Independence of $X$ and the remaining random quantities then enables to proceed as in the proof of Theorem 1 to obtain the result.

\section{A.6. Proof of Theorem 6.}

A.6.1. Proof of part (i). For $t \in[0,1]$ and $i \geq 1$, denote $a_{i}(t)=\left|X_{i}(t)-\widetilde{X}_{i}(t)\right|$. Using the argumentation as in the proof of Bott et al. (2013, Theorem 1) it can be shown that for any $\varepsilon>0$

$$
\sup _{u \in \mathbb{R}}\left|\widetilde{F}_{n, t}(u)-F_{t}(u)\right| \leq \frac{1}{\varepsilon n} \sum_{i=1}^{n} a_{i}(t)+\eta_{n}(t)+\sup _{\left|s-s^{\prime}\right| \leq \varepsilon}\left|F_{t}(s)-F_{t}\left(s^{\prime}\right)\right|,
$$

where $\eta_{n}(t)=\sup _{u \in \mathbb{R}}\left|F_{n, t}(u)-F_{t}(u)\right|$ and $\widetilde{F}_{n, t}$ stands for the distribution function of $\widetilde{P}_{n, t} \in \mathcal{P}(\mathbb{R})$. This allows us to write

$$
\begin{aligned}
\sup _{x \in L^{2}([0,1])} & \left|a D\left(x ; \widetilde{P}_{n}\right)-a D(x ; P)\right| \leq \int_{0}^{1} \sup _{u \in \mathbb{R}}\left|F_{t}(u)-\widetilde{F}_{n, t}(u)\right| \mathrm{d} \lambda(t) \\
& \leq \frac{1}{\varepsilon n} \sum_{i=1}^{n} \int_{0}^{1} a_{i}(t) \mathrm{d} \lambda(t)+\int_{0}^{1} \eta_{n}(t) \mathrm{d} \lambda(t)+\int_{0}^{1} \sup _{\left|s-s^{\prime}\right| \leq \varepsilon}\left|F_{t}(s)-F_{t}\left(s^{\prime}\right)\right| \mathrm{d} \lambda(t) .
\end{aligned}
$$

Because (25) holds true for any positive $\varepsilon$, it is also true if we take an infimum over the set $\{\varepsilon>0\}$ on both sides of the inequality. Bounding further the last summand in (25) 
by $(12)$,

$\sup _{x \in L^{2}([0,1])}\left|a D\left(x ; \widetilde{P}_{n}\right)-a D(x ; P)\right| \leq \inf _{\varepsilon>0}\left(\frac{1}{\varepsilon n} \sum_{i=1}^{n} \int_{0}^{1} a_{i}(t) \mathrm{d} \lambda(t)+\delta_{F}(\varepsilon)\right)+\int_{0}^{1} \eta_{n}(t) \mathrm{d} \lambda(t)$.

The first term on the right hand side relates to the Legendre transform (13), as can be seen from

$$
\begin{aligned}
\inf _{\varepsilon>0}\left(\frac{1}{\varepsilon n} \sum_{i=1}^{n} \int_{0}^{1} a_{i}(t) \mathrm{d} \lambda(t)+\delta_{F}(\varepsilon)\right) & =-\sup _{\varepsilon>0}\left(-\frac{1}{\varepsilon n} \sum_{i=1}^{n} \int_{0}^{1} a_{i}(t) \mathrm{d} \lambda(t)-\delta_{F}(\varepsilon)\right) \\
& =-\sup _{\nu>0}\left(-\nu \frac{1}{n} \sum_{i=1}^{n} \int_{0}^{1} a_{i}(t) \mathrm{d} \lambda(t)-g(\nu)\right) \\
& =-g^{*}\left(-\frac{1}{n} \sum_{i=1}^{n} \int_{0}^{1} a_{i}(t) \mathrm{d} \lambda(t)\right) .
\end{aligned}
$$

Thus, we may write

$$
\sup _{x \in L^{2}([0,1])}\left|a D\left(x ; \widetilde{P}_{n}\right)-a D(x ; P)\right| \leq-g^{*}\left(-\frac{1}{n} \sum_{i=1}^{n} \int_{0}^{1} a_{i}(t) \mathrm{d} \lambda(t)\right)+\int_{0}^{1} \eta_{n}(t) \mathrm{d} \lambda(t) .
$$

Using (11) we have for some $C>0$

$$
\begin{aligned}
\mathrm{E} \frac{1}{n} \sum_{i=1}^{n} \int_{0}^{1} a_{i}(t) \mathrm{d} \lambda(t) \leq \mathrm{E} \frac{1}{n} \sum_{i=1}^{n} \sqrt{\int_{0}^{1} a_{i}(t)^{2} \mathrm{~d} \lambda(t)} & =\frac{1}{n} \sum_{i=1}^{n} \mathrm{E}\left\|X_{i}-\widetilde{X}_{i}\right\| \\
& \leq \frac{1}{n} \sum_{i=1}^{n} C m_{i}^{-\beta /(2 \beta+1)}
\end{aligned}
$$

yielding

$$
\frac{1}{n} \sum_{i=1}^{n} \int_{0}^{1} a_{i}(t) \mathrm{d} \lambda(t)=\mathcal{O}_{P}\left(\frac{1}{n} \sum_{i=1}^{n} m_{i}^{-\beta /(2 \beta+1)}\right)
$$

By Lemma 4 from Appendix A.7, (27) implies

$$
-g^{*}\left(-\frac{1}{n} \sum_{i=1}^{n} \int_{0}^{1} a_{i}(t) \mathrm{d} \lambda(t)\right)=\mathcal{O}_{P}\left(-g^{*}\left(-\frac{1}{n} \sum_{i=1}^{n} m_{i}^{-\beta /(2 \beta+1)}\right)\right) .
$$

For the second term on the right hand side of (26), by Lemma 3 from Appendix A.7 we know that

$$
\int_{0}^{1} \eta_{n}(t) \mathrm{d} \lambda(t)=\mathcal{O}_{P}\left(n^{-1 / 2}\right)
$$

The rate of convergence then follows by combination of (26) with (28) and (29). 
A.6.2. Proof of part (ii). The Legendre transform of $g(\nu)=K \nu^{-\alpha}$ takes the form

$$
g^{*}(\nu)=-K(1+\alpha)\left(-\frac{\nu}{K \alpha}\right)^{\alpha /(1+\alpha)} \quad \text { for } \nu<0 .
$$

Formula (26) can then be rewritten as

$$
\begin{aligned}
\sup _{x \in L^{2}([0,1])}\left|a D\left(x ; \widetilde{P}_{n}\right)-a D(x ; P)\right| \leq & K(1+\alpha)\left(\frac{\frac{1}{n} \sum_{i=1}^{n} \int_{0}^{1} a_{i}(t) \mathrm{d} \lambda(t)}{K \alpha}\right)^{\alpha /(1+\alpha)} \\
& +\int_{0}^{1} \eta_{n}(t) \mathrm{d} \lambda(t),
\end{aligned}
$$

which by (27) and (29) provides the assertion, since the term (27) is dominant. To see this, note that for any positive sequence $\left\{a_{n}\right\}_{n=1}^{\infty}$ we have that

$$
\left|\frac{1}{n} \sum_{i=1}^{n} a_{i}\right| \geq \frac{a_{1}}{n}
$$

meaning that Cesàro's means never converge faster than $\mathcal{O}\left(n^{-1}\right)$, see also Lemma 5 in Appendix A.7. Hence, for any $\alpha \in(0,1]$ for some $C>0$

$$
-g^{*}\left(-\frac{1}{n} \sum_{i=1}^{n} m_{i}^{-\beta /(2 \beta+1)}\right)=C\left(\frac{1}{n} \sum_{i=1}^{n} m_{i}^{-\beta /(2 \beta+1)}\right)^{\alpha /(1+\alpha)} \geq C n^{-\alpha /(1+\alpha)} \geq C n^{-1 / 2} .
$$

A.6.3. Proof of part (iii). Apply Lemma 5 from Appendix A.7 to (27) and use part (ii).

A.7. Auxiliary Results for the Proofs from Section 4. Firstly, we state a useful technical lemma concerning the rate of convergence of an integral of marginal distribution functions of $P$. Here, for $t \in[0,1]$ we write $F_{n, t}$ and $\widetilde{F}_{n, t}$ for the distribution functions of the marginal empirical distributions $P_{n, t}$ and $\widetilde{P}_{n, t}$, respectively.

Lemma 3. For any $P \in \mathcal{P}\left(L^{2}([0,1])\right)$

$$
\int_{0}^{1} \sup _{u \in \mathbb{R}}\left|F_{n, t}(u)-F_{t}(u)\right| \mathrm{d} \lambda(t)=\mathcal{O}_{P}\left(n^{-1 / 2}\right) .
$$

Proof. Denote for $t \in[0,1]$ and $n \geq 1$

$$
\xi_{n}(t)=\sqrt{n} \sup _{u \in \mathbb{R}}\left|F_{n, t}(u)-F_{t}(u)\right| .
$$

For the sequence of random processes $\left\{\xi_{n}(t)\right\}_{n=1}^{\infty}$ the Dvoretzky-Kiefer-Wolfowitz inequality (see Dudley, 1999, Section 6.5) gives us that for any $v>0$

$$
\sup _{t \in[0,1]} \mathrm{P}\left(\xi_{n}(t)>v\right) \leq 2 \exp \left(-2 v^{2}\right) .
$$

Using this inequality we can compute

$$
\mathrm{E} \xi_{n}(t)^{2}=\int_{0}^{\infty} \mathrm{P}\left(\xi_{n}(t)^{2}>v\right) \mathrm{d} \lambda(v) \leq 1 \quad \text { for all } n \geq 1, t \in[0,1] .
$$

By the classical Donsker's theorem for the empirical cumulative distribution function processes (see Dudley, 1999, Theorem 1.8) we know that for any $t \in[0,1]$ the sequence of measures $\left\{\xi_{n}(t)\right\}_{n=1}^{\infty} \subset \mathcal{P}(\mathbb{R})$ converges weakly to a measure in $\mathcal{P}(\mathbb{R})$. This, together with (31) enables us to use a general result of Grinblat (1976, Theorem 3), see also Ivanov (1980, Theorem 1), stating that for any continuous functional $\phi: L^{1}([0,1]) \rightarrow \mathbb{R}$ 
the distribution of $\phi\left(\xi_{n}\right)$ converges to the distribution of the corresponding functional of the limit process. Here, of course, $L^{1}([0,1])$ stands for the space of measurable functions over $[0,1]$ whose absolute value is Lebesgue integrable. Applying this result to $\phi(x)=$ $\int_{0}^{1} x(t) \mathrm{d} \lambda(t)$, we obtain that the sequence of random variables $\left\{\int_{0}^{1} \xi_{n}(t) \mathrm{d} \lambda(t)\right\}_{n=1}^{\infty}$ is tight in $\mathcal{P}(\mathbb{R})$, and the assertion follows.

Lemma 4. Let the functions $\delta_{F}, g$ and $g^{*}$ be defined as in Section 4.2, and let $\left\{a_{n}\right\}_{n=1}^{\infty}$ and $\left\{A_{n}\right\}_{n=1}^{\infty}$ be sequences of positive numbers and random variables, respectively. If $A_{n}=\mathcal{O}_{P}\left(a_{n}\right)$, then $-g^{*}\left(-A_{n}\right)=\mathcal{O}_{P}\left(-g^{*}\left(-a_{n}\right)\right)$.

Proof. Denote $h(\mu)=-g^{*}(-\mu)$, and recall that for any $\mu>0$

$$
h(\mu)=\inf _{\varepsilon>0}\left(\frac{\mu}{\varepsilon}+\delta_{F}(\varepsilon)\right) .
$$

First we show that the function $h$ is non-decreasing over $(0, \infty)$. Fix $0<\mu \leq \mu^{\prime}<\infty$. Then for any $\varepsilon>0$

$$
\frac{\mu}{\varepsilon}+\delta_{F}(\varepsilon) \leq \frac{\mu^{\prime}}{\varepsilon}+\delta_{F}(\varepsilon)
$$

and by taking infimum over all $\varepsilon>0$ we get $h(\mu) \leq h\left(\mu^{\prime}\right)$.

Now, consider $M \geq 1$ and take $\mu>0$ and $\varepsilon>0$ arbitrary. By similar argumentation,

$$
\inf _{\varepsilon>0}\left(\frac{\mu}{\varepsilon}+\frac{\delta_{F}(\varepsilon)}{M}\right) \leq \inf _{\varepsilon>0}\left(\frac{\mu}{\varepsilon}+\delta_{F}(\varepsilon)\right)=h(\mu) .
$$

Multiplying the last inequality by $M$, we get

$$
h(M \mu)=\inf _{\varepsilon>0}\left(\frac{M \mu}{\varepsilon}+\delta_{F}(\varepsilon)\right) \leq M h(\mu) \quad \text { for all } \mu>0, M \geq 1 .
$$

Now, assume that $A_{n}=\mathcal{O}_{P}\left(a_{n}\right)$, i.e. for any $\varepsilon>0$ there exists $C>0$ such that

$$
1-\varepsilon \leq \mathrm{P}\left(A_{n} \leq C a_{n}\right) \leq \mathrm{P}\left(A_{n} \leq \max \{C, 1\} a_{n}\right) \quad \text { for all } n \geq 1 .
$$

Since $h$ is non-decreasing and (32) is true, we may write for $M=\max \{C, 1\}$

$$
\mathrm{P}\left(A_{n} \leq M a_{n}\right) \leq \mathrm{P}\left(h\left(A_{n}\right) \leq h\left(M a_{n}\right)\right) \leq \mathrm{P}\left(h\left(A_{n}\right) \leq M h\left(a_{n}\right)\right),
$$

which enables us to conclude

$$
\mathrm{P}\left(h\left(A_{n}\right)>M h\left(a_{n}\right)\right)<\varepsilon \quad \text { for all } n \geq 1 .
$$

Follows a technical lemma stating the rate of convergence of the sequence of Cesàro's means of a given deterministic sequence. This will be utilized in the proof of Theorem 6 .

Lemma 5. For $r \in \mathbb{R}$ and a sequence $a_{n}=\mathcal{O}\left(n^{r}\right)$ as $n \rightarrow \infty$, consider a sequence $b_{n}=1 / n \sum_{j=1}^{n} a_{j}, n \geq 1$. Then as $n \rightarrow \infty$

$$
b_{n}= \begin{cases}\mathcal{O}\left(n^{r}\right) & \text { if } r>-1, \\ \mathcal{O}(\log (n) / n) & \text { if } r=-1, \\ \mathcal{O}\left(n^{-1}\right) & \text { if } r<-1 .\end{cases}
$$


Proof. By assumptions, there exists $c>0$ such that for all $n$ large enough $\left|a_{n}\right| \leq c n^{r}$. We can write

$$
\left|b_{n}\right| \leq \frac{1}{n} \sum_{j=1}^{n}\left|a_{j}\right| \leq \frac{c}{n} \sum_{j=1}^{n} j^{r} .
$$

Now, it suffices to bound the sum in the last expression, and the desired result follows by application of Euler's summation formula (see Apostol, 1976, Theorem 3.2). 